\title{
Paisaje cultural y evidencias estratigráficas del antropoceno en la Mixteca alta, Oaxaca
}

\author{
Berenice Solís-Castillo, Gonzalo Fernández, Gabriel Vázquez-Castro, Gabriela García-Ayala, \\ Gerardo Bocco, Mario Arturo Ortíz ${ }^{\dagger}$
}

\begin{abstract}
Berenice Solís-Castillo bsolis@ciga.unam.mx

Consejo Nacional de Ciencia y Tecnología, Centro de Investigaciones en Geografía Ambiental, Universidad Nacional Autónoma de México, Antigua Carretera a Pátzcuaro No. 8701, Ex-Hacienda de San José de la Huerta, C.P. 58190, Morelia, Michoacán.
\end{abstract}

\section{Gonzalo Fernández}

Posgrado en Geografía, Instituto de Geografía, Universidad Nacional Autónoma de México, Ciudad Universitaria, Ciudad de México, 04510, México.

\section{Gabriel Vázquez-Castro}

Escuela Nacional de Estudios Superiores Morelia, Universidad Nacional Autónoma de México, Antigua Carretera a Pátzcuaro No. 8701, Col. Ex-Hacienda de San José de la Huerta, C.P. 58190, Morelia, Michoacán, México.

\section{Gabriela García-Ayala}

Posgrado en Manejo Integrado del Paisaje, Centro de Investigaciones en Geografia Ambiental, Universidad Nacional Autónoma de México, Antigua Carretera a Pátzcuaro No. 8701, Col. Ex-Hacienda de San José de la Huerta, C.P. 58190, Morelia, Michoacán, México.

\section{Gerardo Bocco}

Centro de Investigaciones en Geografia Ambiental, Universidad Nacional Autónoma de México, Antigua Carretera a Pátzcuaro No. 8701, Col. Ex-Hacienda de San José de la Huerta, G.P. 58190, Morelia, Michoacán, México.

\section{Mario Arturo Ortíz $\mathbf{z}^{\dagger}$}

Instituto de Geografía, Universidad Nacional Autónoma de México Ciudad Universitaria, Ciudad de México, 04510, México.

BOL. SOC. GEOL. MEX. 2018

VOL. 70 NO. 1

P. $147-171$

http://dx.doi.org/10.18268/BSGM2018v70nla9

Manuscrito recibido: Febrero 15, 2017 Manuscrito corregido: Abril 24, 2017 Manuscrito aceptado: Agosto 31, 2017

\section{RESUMEN}

Se analiza la época propuesta de Antropoceno relacionada con el control de geosistemas por actividades humanas con base en estudios ambientales vs. condiciones antrópicas del desarrollo del suelo en terrazas agrícolas (conocido localmente como lamabordos) en la Mixteca Alta, Oaxaca, México. La compleja relación entre la conformación del paisaje, los cambios en las condiciones ambientales y las transformaciones producto de las transformaciones culturales, constituyen indicadores de la transformación del paisaje a nivel regional de forma espacial y temporal. Estos indicadores conformados por suelos antiguos fácilmente reconocibles y diferenciables por su morfología, coloración, posición estratigráfica y su edad, son producto de las transformaciones culturales controladas por las actividades humanas, vinculadas al aprovechamiento de recursos, el uso de la tierra, la demografía y el ordenamiento territorial de sus asentamientos.

Los suelos más antiguos, chromic Cambisols (calcaric), se formaron durante el Pleistoceno tardío. Durante el Holoceno temprano un incremento de la dinámica erosiva permitió la formación de suelos poco desarrollados (calcic Fluvisol). Los suelos desarrollados a partir de 7900 AP presentan evidencias de impacto antrópico (p.ej. concentración de partículas de carbón). Hacia el Holoceno medio (aproximadamente 5500 AP), la transformación del paisaje toma lugar con la construcción de lamabordos y un aumento en la actividad agrícola; el suelo de esta edad es fácilmente reconocible en campo por lo que se considera un marcador estratigráfico que permite ubicar temporalmente, definir e identificar una nueva época, proponiéndose como el antropoceno en la Mixteca alta. Los suelos del Holoceno tardío, desarrollados entre los 2000 y 500 AP, con mayor contenido de fragmentos de carbón y un aumento en las fases de sedimentación aluvial, indican un uso más intensivo por parte de las sociedades asentadas en la región.

Palabras clave: suelos, agricultura, terrazas, contenido de carbón, actividad humana.

\section{ABSTRACT}

The proposed Anthropocene epoch related to the control of geosistems by human activity is analyzed on the basis of environmental vs. anthropical conditions of soil development on agricultural terraces (known locally as lamabordos) at Mixteca Alta, Oaxaca, Mexico. The complex relationships between landscape development, changes in environmental conditions, and culturally-driven transformations constitute spatial and temporal indicators of landscape transformation at a regional level. These indicators formed of old soils easily recognizable and differentiated by their morphology, color, stratigraphic position, and age, are a result of cultural transformations controlled by human activities, linked to the use of resources, the land usage, demography and land use planning of their settlement.

The older soils, chromic Cambisols (calcaric), were formed during late Pleistocene. During early Holocene an increase of erosional processes allow a poor soil development (calcic Fluvisol). The soils developed 7900 years BP show evidence of anthropic impact (e.g. concentration of carbon particles). Towards middle Holocene (approximately 5500 years BP), the landscape was thoroughly transformed as a consequence of lamabordos building and an increase of the agricultural activity; soils developed are easily recognized in the field and can be regarded as stratigraphic markers. Here we suggest that these soils allow us to link to a new epoch in the Mixteca defined as the Anthropocene. Soils of late Holocene, developed between 2000 and 500 years BP, contain a higher content of carbon fragments and evidence of an increase in the phases of alluvial accumulation, suggesting a more intensive cultural use by settlement groups of the region.

Keywerds: soils, agriculture, terraces, carbon content, human activity. 


\section{Introducción}

El concepto de Antropoceno, definido como etapa donde se produce el control por actividad humana sobre los geosistemas (Vitousek et al., 1997; Crutzen, 2002), ha generado un debate en torno a la capacidad de las sociedades humanas para transformar el clima, el paisaje y sus recursos (Redman, 2004). Muchas disciplinas como la Arqueología, entre otras, se han enfocado al análisis de la definición e implicaciones del término Antropoceno, dirigiendo su atención sobre cómo los seres humanos han transformado el planeta Tierra, así como la relación de los problemas ambientales modernos con el impacto humano a través del tiempo. Desde los años ochenta, se han documentado cambios significativos en el paisaje debido a las actividades humanas. El surgimiento de las sociedades agrícolas tuvo una incidencia importante sobre los ecosistemas reduciendo la diversidad y eficacia de las comunidades naturales. Las prácticas de agricultura en América Latina, particularmente en Centroamérica datan de más de 4000 años AP, en Perú se han hallado zonas cultivadas de 1550 años AP y el cultivo de Yuca en Sudamérica inicia hace 950 años AP (en la costa noroccidental de Lago Maracaibo y en el Bajo Orinoco (Vitale, 1983). También a una escala global ha sido posible distinguir el efecto de la perturbación antrópica en el ambiente en relación a las innovaciones culturales y tecnológicas; tal relación ha permitido el análisis de los cambios en la historia humana, una de esas grandes divisorias es la llamada revolución neolítica o agrícola (Wallerstein, 1979; Osorio, 2015). Los trabajos en Rapa Nui (Hunt y Lipo, 2009), han evidenciado a partir del análisis polínico, una disminución en la taxa de bosque y palmeras, que desaparece alrededor de los 300 años BP y provoca erosión de los suelos volcánicos como lo muestran las partículas de carbón en sedimentos de las excavaciones arqueológicas. Es así que las actividades humanas conducen a un "ecocidio" relacionado con el decaimiento social y la transformación cultural del paisaje. Asimismo, Kirch (2005) además de Erlandson y Rick (2010), con base en los episodios de erosión-sedimentación y datos arqueobotánicos, proporcionaron evidencias de cómo las sociedades cazadoras-recolectoras, pastoriles y agrícolas, han transformado los ecosistemas terrestres y marinos en la Costa del Pacifico por milenios.

Estudios recientes en Mesoamérica han considerado marcadores culturales para señalar cambios ambientales, incluso, se ha establecido la gama de tareas económicas, las que no se distribuyen de forma homogénea en el territorio, y las consecuencias ecológicas de acumulación de excedente en las economías mundo, particularmente, del Posclásico (Williams, 2004). Demostrando con ello la complejidad del cambio ambiental, el rol de las sociedades para influir en tales cambios y respondiendo a ellos desde hace por lo menos 3000 años antes del presente (AP).

El estudio sobre el colapso Maya ha traído consigo un debate en torno a cómo el impacto antrópico sobre el ambiente detonó una transformación cultural, principalmente durante el periodo Clásico $(1300$ - 950 BP), esta importante transformación del medio por el impacto cultural en la región desde el Holoceno medio, ha sido estudiada con diferentes indicadores como registros lacustres (Gill, 2000; Brenner et al., 2001; Rosenmeier et al., 2002; Haug et al., 2003; Hodell et al., 2005a, 2005b; Carleton et al., 2014; Medina-Elizalde et al., 2016) y paleopedológicos (Fedick et al., 2008; Beach et al., 2011; Flores-Delgadillo et al., 2011; Solís-Castillo et al., 2013a, 2013b, 2015; Vázquez et al., 2016), para identificar las modificaciones culturales en el paisaje, particularmente aquellas derivadas de las prácticas agrícolas que han provocado un incremento en la erosión-sedimentación (Dahlin et al., 1980; Dunning et al., 2002; Beach et al., 2003; Dunning et al., 2006; Kennett y Beach, 2013; Liendo et al., 2014; Solís-Castillo et al., 2013a).

En el centro de México, la caída de la ciudad de Teotihuacán tiene una estrecha relación entre el aumento en la deforestación, la degradación evidenciada por el aumento en la sedimentación, poco desarrollo-pérdida del suelo y la desestabilidad social (McGlung et al., 2013; Sánchez-Pérez 
et al., 2013). Otra de las regiones mesoamericanas ampliamente estudiadas es La Mixteca Alta en el sureste de México; los paisajes semiáridos de esta región han sido modificados debido a la creación de grandes centros urbanos en asociación con sistemas agrícolas intensivos. Desde hace 4000 AP se han desarrollado sofisticados sistemas de terrazas que han contribuido en mantener de forma eficiente el control de la erosión y preservado el suelo, aunque en algunas fases de intensificación agrícola los eventos erosivos se incrementan (Kirkby, 1972; Mueller et al., 2012). Las planicies aluviales de Río Verde fueron modificadas debido a la intensificación agrícola, modificando los meandros del río (Goman et al., 2010); en los sedimentos lacustres de la laguna Pastoria, la disminución y aumento en la concentración de partículas de carbón hace referencia a los periodos de ocupación y abandono de los asentamientos humanos (Goman et al., 2013).

Es así, que los procesos de antropización pueden ser identificados en términos temporales y espaciales, siendo posible distinguir en el paisaje las modificaciones culturales de las actividades antrópicas a lo largo del tiempo. La huella antrópica, como se ha mencionado, se reconoce en los registros estratigráficos, por lo que constituye un marcador temporal en el que es posible distinguir cambios generados por el hombre a través del tiempo y sus drásticas repercusiones que han producido una transformación de los ecosistemas y la sociedad.

En el presente trabajo, se revisa el concepto de Antropoceno con base en estudios pedoestratigráficos en terrazas agrícolas de la Mixteca Alta, con la finalidad de ser considerado como época en la escala del tiempo geológico. El objetivo es representar espacial y temporalmente las transformaciones culturales sobre el paisaje, ya que éstas ofrecen una clave para evaluar las interacciones entre las actividades humanas, el ambiente y los cambios culturales que se emplazan sobre el paisaje a una escala regional desde hace $c a .5000$ AP; el análisis se realiza con base en la reconstrucción de las condiciones ambientales de desarrollo de suelo y su comparación con la conformación de suelos que son producto de la transformación cultural e intervención antrópica en las etapas de ocupación humana en la Mixteca Alta. Dichas transformaciones, se propone, están controladas por las actividades humanas vinculadas al aprovechamiento de recursos, el uso de la tierra, la demografía y el ordenamiento territorial de sus asentamientos.

\section{2. Área de estudio}

El área de estudio se localiza en la cuenca del río Yanhuitlán que forma parte del valle de Nochixtlán, en la región de La Mixteca Alta (Figura 1). La Mixteca Alta es una región geográficamente diversa ubicada al noroeste del estado de Oaxaca, colindando con los estados vecinos de Puebla y Guerrero, en el sureste de México (Palacio-Prieto et al., 2016). Es la zona de contacto entre la Sierra Madre del Sur y la Sierra Madre Oriental, con una topografia de montañas medias y valles amplios con laderas fuertemente erosionadas, las cuales aportan gran cantidad de sedimentos al fondo de valle.

La litología de la cuenca del río Yanhuitlán comprende rocas ígneas y sedimentarias, con edades desde el Paleozoico al Cuaternario y diferentes ambientes de formación, lo cual influye de manera decisiva en las formas del terreno y en los procesos erosivos pasados y actuales. Yanhuitlán es una depresión estructural que se formó por el fallamiento de la cubierta sedimentaria; está delimitada hacia el poniente por los bloques tectónicos e ígneos intrusivos asociados al sistema de fallas Cieneguillas (Ferrusquía, 1970) y hacia el oriente por la disposición en cuesta de la Formación Yanhuitlán.

La Formación Yanhuitlán aporta la mayor parte de sedimentos a las terrazas objeto del presente estudio. Ésta se compone de una sucesión de estratos rítmicos finos, arcillo-limosos amarillo-rojizos, con una profundidad de $300-600 \mathrm{~m}$. Sus sedimentos fueron depositados en ambientes lacustres durante el Paleoceno-Eoceno con edad estimada a partir de fechamientos de las intrusiones magmáticas dentro de la misma en $40.5 \pm 1.7 \mathrm{Ma}$ (Martiny et 




Figura 1 Localización del área de estudio. a) muestra la ubicación en la República Mexicana y b) en relación a la ciudad de Oaxaca mostrando la configuración del relieve. c) muestra el sitio de muestreo dentro del municipio de Yanhuitlán. En el extremo inferior derecho de la figura se indican las coordenadas geográficas del sitio de muestreo.

al., 2000). Geomorfológicamente está constituida por lomeríos alargados, redondeados en sus partes altas y con laderas profundamente disectadas. Los arroyos forman valles estrechos y profundos cañones en los que existe un proceso de erosión regresiva. La disposición casi horizontal de los estratos da origen a un sistema de drenaje local de tipo dendrítico y ocasionalmente radial alrededor de algunos intrusivos que aparecen sobre esta formación (Schlaepfer, 1976), sin embargo, a nivel regional se observa un drenaje primordialmente asimétrico (Figura 2).

Dentro de la Formación Yanhuitlán encontramos terrígenos con tamaños de partículas diferentes según su ambiente de depósito. Schlaepfer (1970) distingue dos zonas: la parte central de la cuenca donde los tamaños son predominantemente finos, con proporciones casi iguales entre limos y arcillas y un contenido de arena menor al $10 \%$ debido a que fueron depositados en un ambiente de energía baja y constante; las márgenes de la cuenca presenta por otro lado la mayor frecuencia de horizontes arenosos, con una variación cíclica que indica transporte por agentes de energía variable e intercalada.

El clima de la cuenca es templado subhúmedo 


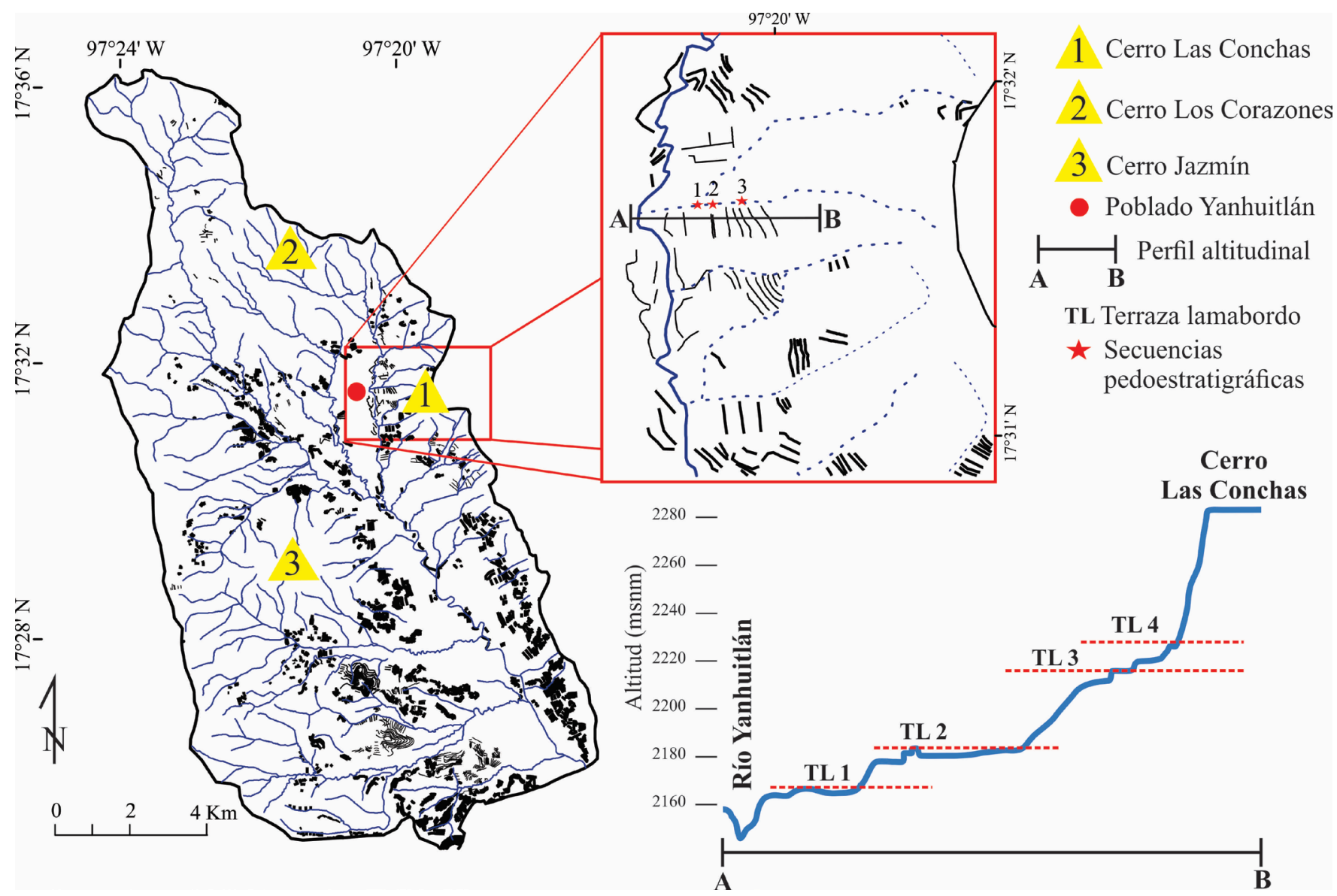

Figura 2 a) Distribución espacial de las terrazas culturales en la cuenca del río Yanhuitlán y su relación con el drenaje, b) ubicación de las secuencias pedoestrtigráficas analizadas en el presente estudio y su relación con las terrazas agrícolas, y c) perfil topográfico de las terrazas culturales asociadas a los puntos de muestreo.

con lluvias concentradas en verano $(\mathrm{Cw})$ y periodo de canícula marcado entre julio-agosto (García, 2004), con temperatura media anual de $15^{\circ} \mathrm{C}$ y precipitación media anual de $649 \mathrm{~mm}$ (estación climática de Yanhuitlán). La vegetación predominante es el chaparral y pastizal inducido con algunos relictos de bosque de Táscate en la parte baja e intermedia de la cuenca del río Yanhuitlán y bosque de encino-pino secundario en las partes altas de la cuenca, con algunos relictos primarios. Ésta es en su mayoría secundaria debido al intenso manejo y cambio de uso del suelo por agricultura y ganadería, en especial el pastoreo.

\subsection{HISTORIA GULTURAL DE LA MIXTEGA ALTA}

La Mixteca Alta oaxaqueña es una zona con altas montañas en donde la morfología plana es escasa. Uno de los principales valles y el más extenso es el de Nochixtlán, en el cual se establecieron diversos asentamientos humanos desde hace más de tres mil años. El valle está dividido en cuatro secciones (brazos): sección noroeste o Yanhuitlán; sección norte o Yucuita; sección suroeste o Quilintongo y sección este o Sayultepec/Etlatongo (Spores, 1972).

La distribución de los asentamientos fue dinámica en tiempo y espacio, pero con claros patrones de 
estabilidad y de ubicación. En el periodo Preclásico temprano y medio (3150 - 2150 años BP; fase regional Cruz) los principales pueblos se ubicaron al norte y centro del valle de Nochixtlán, generalmente localizados en las laderas bajas de los cerros y cercanos a fuentes de agua como arroyos y manantiales (Spores, 1972; Zárate, 1987).

En el Preclásico tardío (200/250 AC - 250/300 DC; fase regional Ramos) comienza un proceso de urbanización, explosión demográfica y complejidad en las relaciones sociales, además de un cambio abrupto en la ubicación de los principales asentamientos hacia las cimas de los cerros (Spores, 1972; Kowalewski et al., 2009). En Yucuita se concentró el poder político y comercial de gran parte del valle de Nochixtlán. A lo largo de la ciudad se ubicaron sitios de menor rango político como aldeas y rancherías, además de gran cantidad de terrazas agrícolas en las laderas de los cerros (Spores, 1972; Plunket, 1990).

La complejidad y la densidad poblacional aumenta durante el periodo Clásico (1650 - 1000 BP.; fase regional: Las Flores); calculando un promedio de 19758 personas para esta fase que comparada con la fase anterior (Ramos; con una población promedio de 6008 habitantes) aumenta a más del doble (Kowalewski et al., 2009).

Durante el Clásico las redes comerciales aumentaron, se comerciaba con el valle de Oaxaca, Veracruz y Puebla (Plunket, 1990; García, 2011), y se desarrollaron nuevos y más importantes centros urbanos. El sitio dominante fue Yucuñudahui, el posible sucesor de Yucuita, pero existieron otros sitios de poder político menor, o centros secundarios como Topiltepec y Perales (Spores, 1972; Heredia, 2007; Kowalewski et al., 2009).

La Mixteca tiene su apogeo durante el periodo Posclásico (1050 - 429 BP) fase regional Natividad); en este periodo existe un importante incremento poblacional y se desarrolla una agricultura intensiva (Spores, 1967, 1972; Romero, 1996; Lind, 2008). En el valle de Nochixtlán, en este periodo se produce una menor inversión en la construcción de edificios cívico-ceremoniales y políticos, pero una mayor inversión de tiempo en la construcción y mantenimiento de terrazas agrícolas y lamabordos. A pesar de que gran parte de los terrenos del valle de Nochixtlán fueron utilizados para vivir y sembrar, algunos asentamientos presentaron algunas edificaciones con fines políticos, otorgándole una mayor jerarquía en la organización sociopolítica de ese momento. Etlatongo y Yucuita retoman su pasado de asentamiento de centros políticos y surgen los sitios de Chachoapan y Nochixtlán, también como posibles centros políticos secundarios. Pero los mayores cambios sucedieron en el valle de Yanhuitlán, el cual tuvo el mayor número de asentamientos y probablemente el control de la mayoría de los asentamientos establecidos en el valle de Nochixtlán (Spores, 1972).

\section{Materiales y métodos}

Se realizaron estudios detallados de la pedoestratigrafía del Holoceno en el noroeste del valle de Nochixtlán, a lo largo del río Yanhuitlán. Seis secciones estratigráficas localizadas en las coordenadas $17^{\circ} 31^{\prime} 48.02^{\prime \prime} \mathrm{N}, 97^{\circ} 20^{\prime} 17^{\prime \prime} \mathrm{W}$ a una altitud de 2149 msnm, que están asociadas a terrazas culturales fueron descritas y muestreadas: YAN 01 a YAN 06 (Figura 2a y b). Se reconocieron también las terrazas aluviales y culturales (lamabordos y terrazas de contorno), sobre las cuales se desarrollaron los suelos antrópicos que fueron fechados por radiocarbono, permitiendo con ello establecer las edades del paisaje cultural (Figura 2c).

\subsection{ANÁLISIS PEDOESTRATIGRÁFICO: MEMORIA DEL SUELO}

Los suelos antiguos fueron descritos siguiendo los criterios establecidos por la Unión Internacional de la Ciencia del Suelo (IUSS por sus siglas en inglés) (IUSS Working Group WRB. 2015; Retallack, 1990). La descripción de las características morfológicas de los suelos en cada sección fue realizada con base en la identificación de los suelos sepultados y sus horizontes diagnóstico. Los análisis de laboratorio se enfocaron en caracterizar las propiedades asociadas a las características del 
suelo que se conservan a través del tiempo; estas permitieron la formación del suelo y brindan la posibilidad de identificar tanto las características ambientales como aquellas derivadas de las transformaciones antrópicas. La distribución del tamaño de grano, la susceptibilidad magnética y el conteo de partículas de carbón son métodos posibles de ser relacionados con las fases de estabilidad e inestabilidad del paisaje, que pueden derivar tanto de un cambio en la precipitación como un aumento de la perturbación antrópica en la cuenca. El contenido de carbonatos se relaciona con las condiciones ambientales presentes durante la formación del suelo.

La granulometría permite identificar los ciclos de formación de suelo y detectar las discontinuidades. La fracción arena $(2-0.63 \mathrm{~mm})$ fue separada por medio de un tamiz no. 120, la fracción limo $(0.63-0.002 \mathrm{~mm})$ y arcilla $(<0.002 \mathrm{~mm})$ se separó por medio de las diferencias en los tiempo de sedimentación de las partículas y por el método de la pipeta. El contenido de carbonatos fue determinado por pérdida de peso después de la disolución con $0.5 \mathrm{ml}$ de HCl. Las muestras se secaron a 105 ${ }^{\circ} \mathrm{C}$ en un horno mecánico Red Line Blinder durante $72 \mathrm{~h}$, el porcentaje fue calculado usando la diferencia de peso.

La susceptibilidad magnética $(\chi)$ nos permite diferenciar los horizontes de suelo y detectar la presencia de sedimentos en secuencias edafosedimentarias (Vázquez et al., 2016); ésta se midió a lo largo de todas las secciones a intervalos de $10 \mathrm{~cm}$. Las muestras fueron puestas en portamuestras de acrílico no magnético de $8 \mathrm{~cm}^{3}$ para medir la susceptibilidad en alta y baja frecuencia, por medio de un susceptibilímetro Bartington MS2B de sensor dual.

La pedoestratigrafía fue establecida usando fechamientos de ${ }^{14} \mathrm{C}$, evidencias arqueológicas, culturales y la morfología del suelo. Las edades de radiocarbono se obtuvieron de fragmentos de carbón contenidos en los horizontes A en los laboratorios ICA, en Miami, Florida, E.U. Las dataciones están reportadas en edades calibradas de acuerdo al programa CALIBRadiocarbon Calibration 7.0 (Stuiver y Reimer, 1993, 2016).

\subsection{PAISAJE GULTURAL}

La reconstrucción del paisaje cultural se realizó a partir de la delimitación de las geoformas antrópicas (diferentes niveles de terraza, Figura 3), modificando los criterios establecidos por Tricart y Cailleux (1972), considerando patrones morfo-antrópicos espacialmente distribuidos. De esta manera, se aplicaron cuatro criterios principales: a) génesis, b) configuración y arreglo de las geoformas antrópicas, c) procesos de modelado del
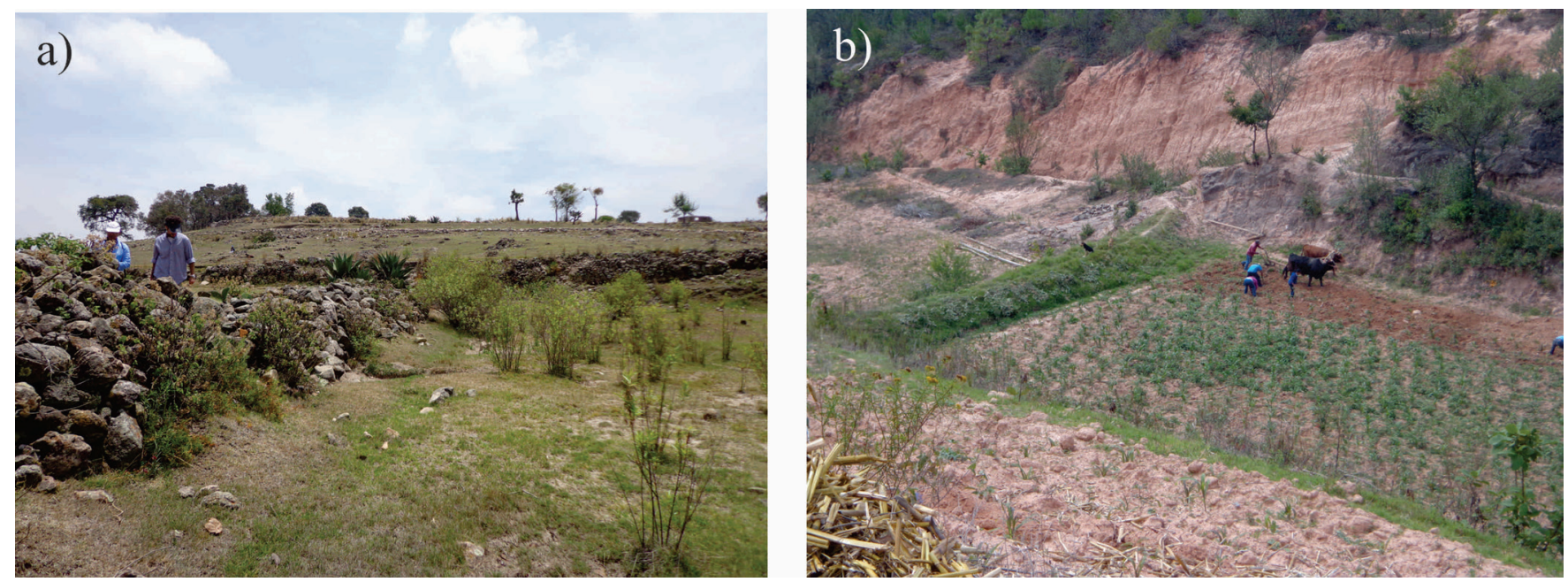
relieve, y d) temporalidad (edades de las estructuras y formas). El control temporal de los patrones morfo-antrópicos se basó en la pedoestratigrafía, fechamientos de ${ }^{14} \mathrm{C}$ y en los estudios estratigráficos de Mueller et al., 2012.

\section{Resultados}

\subsection{PALEOSUELOS ALUVIALES EN ELRÍO YANHUITLÁN}

La sección pedoestratigráfica Yanhuitlán (Figuras 4 y 5 a) está constituida por los seis perfiles mencionados anteriormente. El perfil Yan 01 se compone de los horizontes 17Bw/17Bwg; ambos horizontes se caracterizan por un color pardo rojizo 7.5 YR 4/6, presencia de concreciones de carbonatos, facetas de fricción y moteados de color pardo claro (7.5 YR 3/6), presenta baja susceptibilidad magnética (Figura 4); el espesor del perfil es de 120 cm (Tabla 1). En esta secuencia no se localizaron horizontes A, lo que se interpreta como resultado de procesos erosivos.

El perfil Yan 02 (Figura 5b), se conforma por los horizontes 16Ass/16Bw/16Bk, es también una fase de desarrollo de suelo de pre-ocupación humana, diferenciable en campo por su coloración pardo-rojizo (7.5 YR 5/3); presenta bajo contenido de $\mathrm{CaCO}_{3}$, baja susceptibilidad magnética y un espesor de $172 \mathrm{~cm}$. 16Ass tiene una estructura en bloques angulares, huellas de raíces, color pardo oscuro (10YR 4/1), es duro y compacto, con algunas facetas de fricción y concreciones de carbonatos; se observa un cambio gradual del horizonte $16 \mathrm{Ass}$ al horizonte $16 \mathrm{Bw}$, este último con mayor contenido de arcilla, color pardo-rojizo (7.5 YR $5 / 3)$ y carbonatos en la matriz del suelo. El horizonte 16Bk es de color pardo rojizo (7.5 YR 5/2), textura arcillosa con estructura en bloques angulares y prismáticos y se distingue por el tamaño de las concreciones de carbonatos $(\approx 3 \mathrm{~cm})$.

El perfil Yan 03 (Figura 5c), es una secuencia de seis fases de edafogénesis y en la base de los mismos un evento de sedimentación aluvial. Este perfil se compone de ciclos de desarrollo de suelo con una sucesión de horizontes 13A/13AC, 14A/14AC y 15A/15AC, con espesor de $130 \mathrm{~cm}$. El horizonte 13A se caracteriza por presentar acumulación de materia orgánica, estructura en bloques subangulares, moteados pardo claro, huellas de raíces, matriz color pardo oscuro $10 \mathrm{YR} 5 / 2$, textura arcillo-limosa. En el horizonte 13AC disminuyen raíces, se ven fracciones de arena y limo fino, bloques subangulares pequeños, presenta carbonatos; su color es pardo claro 10YR 5/2. El horizonte 14A presenta mayor contenido de materia orgánica, algunos cutanes muy delgados, huellas de raíces, pedacitos de carbón, estructura subangular, mas arcilloso, presenta carbonatos y un color pardo claro 10YR 5/2. En el horizonte 14AC aumenta el contenido de arenas, pocas huellas de raíces, escasos carbonatos en la matriz, bloques subangulares y un color pardo obscuro 10YR 6/3. El horizonte $15 \mathrm{~A}$ es más obscuro que el superior (10YR 5/2), arcilloso, mejor estructura, bloques angulares, huellas de raíces, moteado de color gris, concreciones de carbonatos, se observan algunos cutanes, carbonatos asociados a las huellas de raíces. El horizonte 15AC presenta textura arenosa con pequeñas rocas, pocas huellas de raíces, sin estructura, presencia de carbonatos y color pardo claro 10 YR 5/2.

Los sedimentos aluviales conforman la sección Yan 04 (Figura 5d); en la sección superior (594 - $684 \mathrm{~cm}$ ) presentan textura arenosa masiva con un alto contenido de carbonatos en la matriz, mientras que en la parte media $(684-784 \mathrm{~cm})$ se observa una estratificación laminar; en la zona inferior $(784-824 \mathrm{~cm})$ los sedimentos presentan estratificación cruzada y están conformados principalmente por suelo re-depositado y arenas finas. Las siguientes nueve fases de desarrollo de suelos (numerados del 4 al 12) conforman la secuencia Yan 05 de $478 \mathrm{~cm}$ de espesor (Figura 5e-g). El paleosuelo 12, tiene múltiples horizontes 12A/12B/12C; la matriz del horizonte $12 \mathrm{~A}$ es de color pardo-rojizo (10 YR 5/2) con moteados y concreciones de carbonatos aproximadamente de $4 \mathrm{~cm}$ de diámetro. El horizonte 12B de color pardo-claro (10YR 4/2) presenta estructura en bloques angulares, contie- 


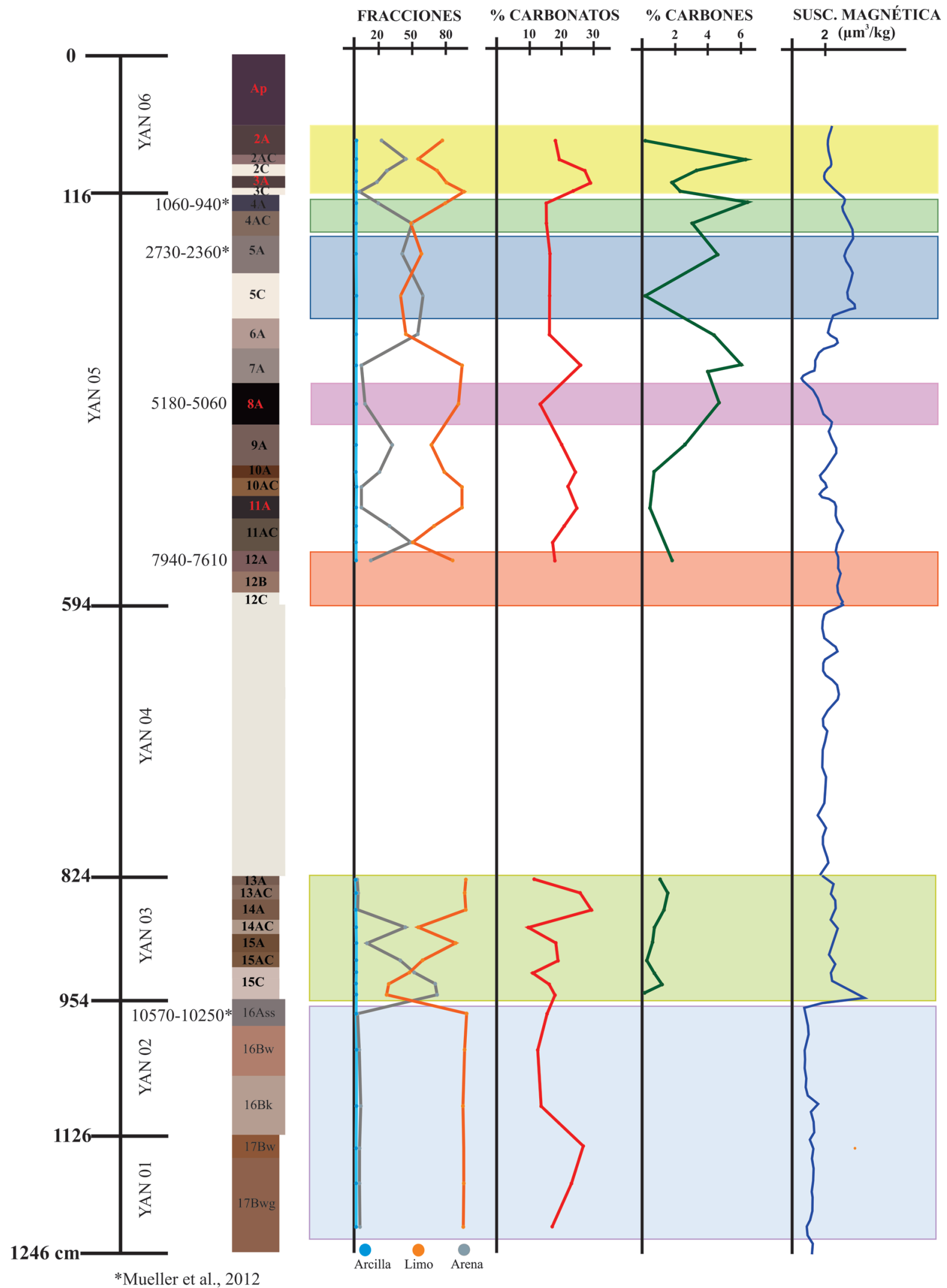


Tabla 1. Rasgos morfológicos. Rasgos morfológicos de la secuencia pedoestratigráfica Yanhuitlán.

\begin{tabular}{|c|c|c|c|}
\hline Sección & Horizonte & $\begin{array}{l}\text { Profundidad } \\
(\mathrm{cm})\end{array}$ & Color \\
\hline \multirow{6}{*}{ Yan 06} & Ap & $0-6$ & $7.5 \mathrm{YR} 5 / 4$ \\
\hline & $2 \mathrm{~A}$ & $6-26$ & 7.5 YR $5 / 4$ \\
\hline & $2 \mathrm{AC}$ & $26-60$ & $7.5 \mathrm{YR} 4 / 3$ \\
\hline & $2 \mathrm{C}$ & $60-85$ & 10YR $5 / 1$ \\
\hline & $3 \mathrm{~A}$ & $85-93$ & 10YR $4 / 3$ \\
\hline & $3 \mathrm{C}$ & $93-103$ & $2.5 \mathrm{Y} 5 / 2$ \\
\hline \multirow{9}{*}{ Yan 05} & $4 \mathrm{~A}$ & $116-151$ & 10 YR $4 / 3$ \\
\hline & $4 \mathrm{AC}$ & $51-170$ & 10YR $5 / 2$ \\
\hline & $5 \mathrm{~A}$ & $170-208$ & 10YR $6 / 2$ \\
\hline & $5 \mathrm{C}$ & $208-256$ & 10YR $6 / 2$ \\
\hline & $6 \mathrm{~A}$ & $256-286$ & 10YR $6 / 2$ \\
\hline & $7 \mathrm{~A}$ & $286-316$ & 10YR 6/1 \\
\hline & $8 \mathrm{~A}$ & $316-356$ & 10YR $4 / 1$ \\
\hline & $9 \mathrm{~A}$ & $356-400$ & 10YR $6 / 2$ \\
\hline & $10 \mathrm{~A}$ & $400-415$ & 10YR $6 / 2$ \\
\hline
\end{tabular}

\section{Edad

Edad

Estructura subangular, agregados de tamaño fino a mediano, friable, gran cantidad de bioporos y carbonatos en la matriz, el límite con el horizonte inferior es difuso. Textura francoarcillosa.

Estructura columnar que rompe a bloques subangulares, mayor estabilidad en los agregados los que van de gruesos a moderados, biporos y huellas de raíces, alta concentración de carbonatos en la matriz.

Estructura columnar que rompe a bloques angulares, mayor estabildad en lo agregados, alta concentración de carbonatos en la matriz.

Estructura angular con fragmentos de roca de $1 / 2 \mathrm{~cm}$, huellas de raíces muy duras, textura limosa, presenta grietas y concreciones de carbonatos y $\mathrm{CaCO}_{3}$ en la matriz, color pardo claro.

Menor desarrollo de estructura, de color pardo, poca materia orgánica, con presencia de carbonatos en la matriz.

Sedimento de color pardo claro, reacción al $\mathrm{HCl}$, con concreciones de carbonatos.

Color gris oscuro, casi negro, moteado color gris, huellas de $1060-940^{*}$ raíces, bioporos, rocas (algunos de $3 \mathrm{~mm}$ ), presenta mucho carbón, algunos cutanes, estructura angular casi columnar.

Sedimento de arenas y algunos cantos rodados con contenido de materia orgánica, sin estructura, parte superior de arena, a los $46 \mathrm{~cm}$ comienzan los cantos rodados de $1-6$ $\mathrm{cm}$ aproximadamente, presenta mucho carbón.

Textura arenosa, presenta mucho carbón, huellas de raíces, raíces rellenas de material arcilloso de color rojizo, estructura en bloques subangulares pequeños y muy frágiles, aumenta partículas de carbón.

Aluvión de cantos rodados de $2-10 \mathrm{~cm}$.

Suelo arenoso, presenta mucho carbón, poroso, presenta carbón en fragmentos pequeños, estructura en bloques pequeños muy friables, color pardo.

Estructura en bloques subangulares, arcilloso, motas de color grisáceo, huellas de raíces, color más oscuro que el limite anterior (café oscuro).
$5300-5190$

$(43.1 \%)$

$5180-5060$

$(52.3 \%)$
Suelo negro, estructura en bloques angulares casi columnares, muy arcilloso, huellas de raíces, facetas de fricción, concreciones de carbonatos.

Estructura en bloques angulares, mas arenoso que el límite anterior, presencia de carbón, concreciones de carbonatos, huella de raíces, presenta más poros.

Estructura en bloques subangulares, poca presencia de carbón, arenoso, color pardo.

* Edades reportadas por Mueller et al., 2012. 
Tabla 1. Rasgos morfológicos. Rasgos morfológicos de la secuencia pedoestratigráfica Yanhuitlán. (Continuación).

\begin{tabular}{|c|c|c|c|c|c|}
\hline Sección & Horizonte & $\begin{array}{l}\text { Profundidad } \\
\quad(\mathrm{cm})\end{array}$ & Color & Edad & Descripción \\
\hline \multirow{6}{*}{ Yan 05} & $10 \mathrm{AC}$ & $415-440$ & 10YR 6/1 & & $\begin{array}{l}\text { Estructura en bloques angulares, pequeños fragmentos de } \\
\text { roca }(4 \mathrm{~mm}) \text {, huellas de raíces, arcilloso, presencia de } \\
\text { concreciones de carbonatos, presencia de carbones. }\end{array}$ \\
\hline & $11 \mathrm{~A}$ & $440-466$ & 10 YR $5 / 2$ & & $\begin{array}{l}\text { Estructura en bloques angulares, mas arenoso que el limite } \\
\text { superior, escasa presencia de carbones, iluviación de } \\
\text { carbonatos en la superficie de los agregados y en los } \\
\text { bioporos, presencia de carbón. }\end{array}$ \\
\hline & $11 \mathrm{AC}$ & $466-433$ & 10YR 6/3 & & $\begin{array}{l}\text { Baja proporción de carbonatos en los bioporos, presencia de } \\
\text { carbones, moteado color gris, color de la matriz del suelo } \\
\text { pardo y oscuro, estructura en bloques columnares. } \\
\text { Sedimentos arenosos, color pardo amarillento, algunos } \\
\text { fragmentos rocosos pequeños }(1 \mathrm{~cm}) \text {, rocas más grandes } 1- \\
4 \mathrm{~cm} \text {. }\end{array}$ \\
\hline & $12 \mathrm{~A}$ & $433-456$ & $10 Y R 5 / 2$ & $7940-7610$ & $\begin{array}{l}\text { Presencia de materia orgánica, estructura en bloques } \\
\text { angulares, huella de raíces rellenas de arcilla de color rojizo, } \\
\text { presencia de carbonatos en superficie de agregados, } \\
\text { moteado de color gris - rojizo, presencia de carbonatos en } \\
\text { fragmentos de tamaño } 4 \mathrm{~cm} \text { aproximadamente. }\end{array}$ \\
\hline & $12 \mathrm{~B}$ & $456-492$ & $10 \mathrm{YR} 4 / 3$ & & $\begin{array}{l}\text { Estructura en bloques angulares, huellas de raíces, } \\
\text { carbonatos, arenoso, color pardo claro. }\end{array}$ \\
\hline & $12 \mathrm{C}$ & $492-594$ & $10 Y R 4 / 2$ & & Paquete sedimentario con un pequeño desarrollo de suelo. \\
\hline \multirow{3}{*}{ Yan 04} & $\mathrm{~S} 1$ & $594-684$ & & & $\begin{array}{l}\text { Sedimentos aluviales de textura arenosa y masivos con un } \\
\text { alto contenido de carbonatos. }\end{array}$ \\
\hline & S2 & $684-784$ & & & Sedimentos aluviales en estratificación laminar. \\
\hline & S3 & $784-824$ & & & $\begin{array}{l}\text { Sedimentos aluviales de arenas finas con estratificación } \\
\text { cruzada y suelo redepositado. }\end{array}$ \\
\hline \multirow{7}{*}{ Yan 03} & $13 \mathrm{~A}$ & $824-845$ & 10YR 5/2 & & $\begin{array}{l}\text { Acumulación de materia orgánica, estructura en bloques } \\
\text { subangulares, moteados pardo claro, huellas de raíces, } \\
\text { matriz color pardo oscuro, textura arcillo-limosa. }\end{array}$ \\
\hline & $13 \mathrm{AC}$ & $845-857$ & 10YR $5 / 2$ & & $\begin{array}{l}\text { Color pardo claro, disminuyen raíces, se ven fracciones de } \\
\text { arena y limo fino, bloques subangulares pequeños, presenta } \\
\text { carbonatos. }\end{array}$ \\
\hline & $14 \mathrm{~A}$ & $857-874$ & $10 Y R 5 / 2$ & & $\begin{array}{l}\text { Mayor contenido de materia orgánica, se ven algunos } \\
\text { cutanes muy delgados, huellas de raíces, hay pedacitos de } \\
\text { carbón, estructura subangular, mas arcilloso, presenta } \\
\text { carbonatos. }\end{array}$ \\
\hline & $14 \mathrm{AC}$ & $874-885$ & 10YR $6 / 3$ & & $\begin{array}{l}\text { Aumenta el contenido de arenas, pocas huellas de raíces, } \\
\text { escasos carbonatos en la matriz, bloques subangulares. }\end{array}$ \\
\hline & $15 \mathrm{~A}$ & $885-910$ & $10 Y R 5 / 2$ & & $\begin{array}{l}\text { Es más obscuro que el superior, arcilloso, mejor estructura, } \\
\text { bloques angulares, huellas de raíces, moteado de color gris, } \\
\text { concreciones de carbonatos, se observan algunos cutanes, } \\
\text { carbonatos asociados a las huellas de raíces. }\end{array}$ \\
\hline & $15 \mathrm{AC}$ & $910-925$ & 10YR $6 / 2$ & & $\begin{array}{l}\text { Textura arenosa con pequeñas rocas, pocas huellas de } \\
\text { raíces, sin estructura, presencia de carbonatos. }\end{array}$ \\
\hline & $15 \mathrm{C}$ & $925-954$ & 10YR 5/2 & & $\begin{array}{l}\text { Sedimento aluvial con estratificación cruzada y presencia de } \\
\text { carbonatos. }\end{array}$ \\
\hline
\end{tabular}


Tabla 1. Rasgos morfológicos. Rasgos morfológicos de la secuencia pedoestratigráfica Yanhuitlán. (Continuación).

\begin{tabular}{|c|c|c|c|c|c|}
\hline Sección & Horizonte & $\begin{array}{l}\text { Profundidad } \\
\text { (cm) }\end{array}$ & Color & Edad & Descripción \\
\hline \multirow{3}{*}{ Yan 02} & 16Ass & $954-989$ & 10YR 4/1 & $\begin{array}{l}10570- \\
10250 *\end{array}$ & $\begin{array}{l}\text { Estructura en bloques angulares, huellas de raíces color } \\
\text { negro pardo oscuro, duro, compacto, algunas facetas de } \\
\text { fricción, estructura angulosa, motas de color pardo rojizo, } \\
\text { concreciones de carbonatos. }\end{array}$ \\
\hline & $16 \mathrm{Bw}$ & $989-1040$ & 7.5 YR $5 / 3$ & & $\begin{array}{l}\text { Cambio gradual de pardo oscuro a pardo claro, moteado } \\
\text { rojizo igual que el superior, concreciones, textura arcillosa, } \\
\text { huellas de raíces. }\end{array}$ \\
\hline & $16 \mathrm{Bk}$ & $1040-1126$ & 7.5 YR $5 / 2$ & $\begin{array}{l}10570- \\
10250^{*}\end{array}$ & $\begin{array}{l}\text { Mayor cantidad de concreciones de carbonatos, color más } \\
\text { oscuro que el superior, con presencia de raíces, facetas de } \\
\text { fricción, textura arcillosa, estructura en bloques angulares y } \\
\text { prismáticos. }\end{array}$ \\
\hline \multirow[t]{2}{*}{ Yan 01} & $17 \mathrm{Bw}$ & $1126-1156$ & 7.5 YR $4 / 4$ & & $\begin{array}{l}\text { Bloques angulares, arcilloso, presencia de raíces, color } \\
\text { pardo oscuro, rellenos de material de suelo, concreciones de } \\
\text { carbonato, facetas de fricción en la superficie de los } \\
\text { agregados, carbonatos en la matriz del suelo, motas color } \\
\text { pardo claro, presencia de carbonatos en los poros y en la } \\
\text { matriz color } 7.5 \text { YR } 4 / 6 \text {. }\end{array}$ \\
\hline & 17Bwg & $1156-1246$ & 7.5YR 4/4 & & $\begin{array}{l}\text { Motas amarillentas, algunos poros, concreciones de } \\
\text { carbonatos, huellas de raíces, facetas de fricción, raíces } \\
\text { rellenas de material de suelo, el moteado se incrementa con } \\
\text { la profundidad, estructura en bloques angulares color rojizo } \\
(7.5 \text { YR 4/6). }\end{array}$ \\
\hline
\end{tabular}

* Edades reportadas por Mueller et al., 2012.

ne menor cantidad de concreciones de carbonatos y mayor cantidad de arcillas; el horizonte $12 \mathrm{C}$ es un paquete sedimentario areno-limoso de color pardo muy claro (10YR 4/2). El paleosuelo 11 presenta a los horizontes $11 \mathrm{~A} / 11 \mathrm{AC}$, tiene un ligero aumento en la concentración de fragmentos de carbón en su horizonte $11 \mathrm{AC}$, con una matriz de color pardo-obscuro (10YR 6/3), pero mayor de carbonatos en el horizonte $11 \mathrm{a}$, que contribuye con el color pardo (10YR 5/2) (Figura 4, Tabla 1). El paleosuelo 10 está conformado por los horizontes 10A/10AC, el horizonte 10A se caracteriza por una matriz de color pardo 10YR 6/2, una estructura en bloques subangulares mientras que el horizonte 10AC por fragmentos de roca, escasos carbones y concreciones de carbonatos, con una coloración pardo claro 10YR 6/1). En el paleosuelo 9A tiene una estructura en bloques angulares y concreciones de carbonatos en la matriz de color pardo (10YR6/2).

El paleosuelo 8A es el más representativo del perfil Yan 05, es un suelo negro (10YR 4/1), con es- tructura en bloques angulares casi columnar, con concreciones de carbonatos y huellas de raíces y presencia de carbón. 7A con una estructura en bloques subangulares con motas de color grisáceo (10YR 6/1) diferente de 6A es poroso, con estructura en bloques angulares pequenos muy friables de color pardo (10YR 6/2); 5A es de textura arenosa, con recubrimientos rojizos en los bioporos tiene una estructura en bloques subangulares pequenos y friables, mientas que $5 \mathrm{C}$ de color $10 \mathrm{YR}$ 6/2 es un aluvión de cantos rodados. El horizonte 4A de color gris obscuro (10YR 4/3), con rasgos reductomórificos como moteados, las huellas de raíces y biporos, cutanes y conuns estructura caso columna, siendo la presencia de rocas su principal característica; el horizonte $4 \mathrm{AC}$ de color pardo calro (10YR 5/2) es un sedimentos de arenosos y algunos cantos rodados.

La sección Yan 06 contiene el suelo reciente, protovertic cambic calcaric PHAEOZEM (clayic) (Ap/AB/ BWk) y los paleosuelos 2A/2AC/2C/3A/3C. El horizonte $2 \mathrm{~A}$ de estructura columnar tienen una 

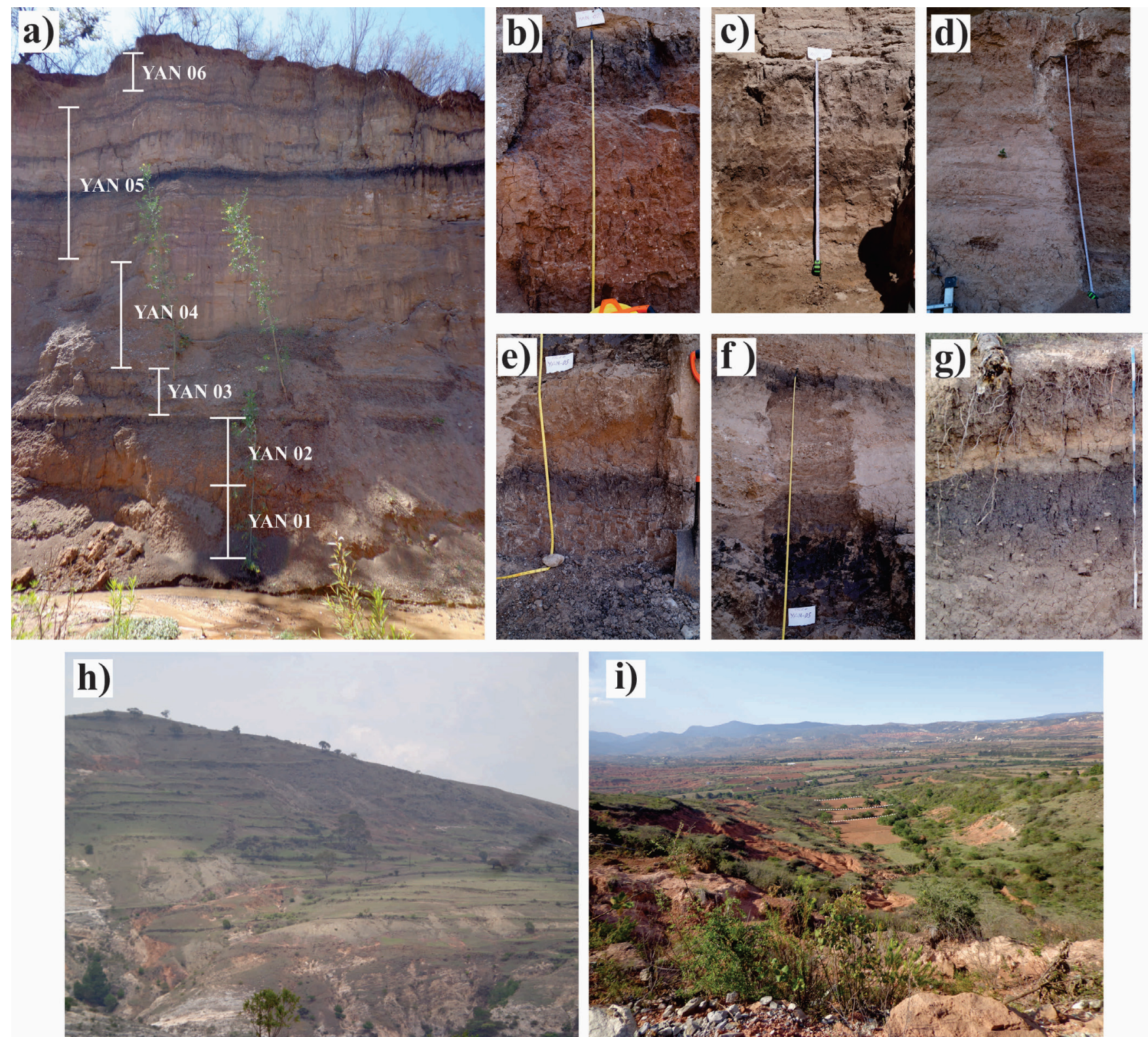

Figura 5 Paleosuelos asociados a terrazas agrícolas., a) Terrazas de contorno, b) Lamabordos, c) Secuencias aluviales en el río Yanhuitlán, d) Suelos pleistoceno, e) Suelos del Holoceno temprano, f) Sedimentos aluviales, g) suelos del Holoceno temprano-medio (aprox. 7000 años AP), h) Suelo desarrollado hace aprox. 5000 años AP (parte inferior de la fotografía) y, i) Suelos con fragmentos de cerámica y concreciones de carbonatos (edad aprox. 1000 años AP).

mayor estabilidad en los agregados, bioporos y con un color de la matriz pardo-griscaceo 7.5YR 5/4; $2 \mathrm{AC}$, de estructura columnar que rompe a bloques angulares, tiene una mayor estabilidad en los agregados y una alta concentración de carbonatos en la matriz de color pardo obscuro (10YR 4/3), 2C se caracteriza por rocas en una matriz de suelo columnar de textura limosa de color pardo claro (10YR 5/1). 3A de color pardo (10YR 4/3) con carbonatos en la matriz del suelo y una estructura poco desarrollada; $3 \mathrm{C}$ de color pardo claro $(2.5 \mathrm{Y} 5 / 2)$ es un sedimento con concreciones de carbonatos. 


\subsection{GARAGTERÍSTICAS ANALÍTICAS}

Los resultados de los análisis de laboratorio nos proporcionan las siguientes propiedades de los suelos (Tabla 1). En Yan 01 aumenta el contenido de limo; el porcentaje de carbonatos es mayor que en Yan 02 (23.2 \%), con una baja susceptibilidad magnética (Figura 4). Yan 02 es ligeramente mas arcilloso aunque en 18Bk incrementa la cantidad de arenas, la susceptibilidad magnética es baja. En Yan 03 aumenta en el contenido de los carbonatos $(19.05 \%)$ y la susceptibilidad magnética. En este paleosuelo se registran carbones macroscópicos $(4.8 \%)$, que conservan una orientación laminar.

La secuencia Yan 05 caracterizada por nueve paleosuelos y es variable en sus características análiticas. Incrementa sustancialmente el contenido de arenas (del 3 al 59\%), las partículas macroscópicas de carbón $(6 \%)$, el contenido de carbonatos varía de 0.3 a $4.3 \%$ y la susceptibilidad magnética aumenta en la sección superior de la secuencia, su rango de valores es de 1 a $10 X \mathrm{~m}^{3} / \mathrm{kg}$. Yan 06 la susceptibilidad magnética disminuye $(5-6.5$ $X \mathrm{~m}^{3} / \mathrm{kg}$ ), las partículas de carbón se incremen$\tan (6 \%)$, los carbonatos varían de 18 a $30 \%$ y la fracción limo es dominante $(50-60 \%)$.

\subsection{GEOMORFOLOGÍA Y TERRAZAS}

En el análisis geomorfológico se identificaron cuatro terrazas de origen antrópico (Figura 2c) con base en su distribución escalonada en planta, presentan una ligera pendiente en dirección W-SW y están asociadas principalmente a la ladera de esa margen del río sin aparente continuidad lateral (Figura 2b). Las terrazas, por su origen, son ambientes acumulativos, regidos por la pendiente, por ende conlleva una sedimentación detrítica que depende de los materiales erosionados, en Yanhuitlán se trata de sedimentos de textura limo-arenosa que de forma continua están sujetos a procesos de erosión y acumulación.

Los cuatro niveles de terraza (Figura 2c) se denominaron desde la más joven; TL1 hasta la más antigua; TL4. Esta última se encuentra a una elevación de 2215 a $2225 \mathrm{msnm}$, con una edad de formación entre los 7900 - 5300 AP; TL3, se encuentra a 2185 - 2215 msnm y se formó aproximadamente entre 5000 - 2730 AP; TL2 se ubica a 2170 - 2185 msnm con una edad de 1060 AP, y finalmente, TL1 se encuentra a $2165-2170 \mathrm{msnm}$ y la que corresponde con la ocupación histórica y reciente. Las terrazas están delimitadas por bordos antrópicos (diques) construidos de forma perpendicular (NW-SE) a la dirección del drenaje del cerro Las Conchas y en las cuales se han centrado los análisis pedoestratigráficos (Figura 2b). Las terrazas culturales (lamabordos) en el valle de Yanhuitlán se distribuyen a lo largo de los cauces, cuestas y laderas de las principales elevaciones de la región como son los cerros El Jazmín, Los Corazones, Loma Taza, Las Canicas y Las Conchas (Figura 2).

\section{Discusión}

\subsection{GÉNESIS Y EVOLUGIÓN DEL PAISAJE}

Establecer la génesis del paisaje y el cambio en las condiciones ambientales, ha sido una práctica que permite reconocer los patrones naturales de modificación del paisaje a través del tiempo, sin embargo, estás herramientas son también de utilidad cuando la finalidad es caracterizar y diferenciar el impacto antrópico ocurrido en los ambientes naturales, durante las etapas más recientes de la historia ambiental de la región.

La escala temporal de referencia en la transformación cultural del paisaje en el valle de Yanhuitlán se ha establecido con base en fechamientos por radiocarbono y utilizando la morfología de paleosuelos y suelos antrópicos. El paleosuelo en la base de la secuencia Yan 02, chromic Cambisol (calcaric), presenta fuertes rasgos de intemperismo, reductomórficos, lixiviación y neoformación de carbonatos (concreciones). Todas estas características evidencian un ambiente húmedo y cálido durante la formación del suelo, aunque la neoformación de carbonatos sugiere un cambio hacia condiciones más áridas, presumiblemente al final del Pleis- 
toceno. Mueller et al. (2012) también propone que en la última etapa del Pleistoceno (Ultimo Máximo Glacial) las condiciones áridas prevalecieron como se reporta en el sureste de México y Guatemala (Leyden, 2002; Lozano et al., 2005; Piperno et al., 2007; Hodell et al., 2008; Ortega et al., 2010). El suelo Yan 02 (chromic Cambisol) pertenece a una edad de 10570 - 10250 AP (años calibrados antes del presente) (Mueller et al., 2012), con fuertes rasgos de intemperismo en sus horizontes $16 \mathrm{Bw}$ refiere a un clima húmedo que cambia hacia un clima estacional debido a la presencia de rasgos de expansión-contracción a lo largo de la secuencia. El depósito de sedimentos aluviales en tres facies que cubren el periodo entre $10570 \mathrm{y} \approx 7800 \mathrm{AP}$ es anterior a la llegada de los primeros agricultores, siendo la dinámica erosiva producto de un cambio en la vegetación a los 9000 AP que ha sido registrado en la Gueva de Guila Naquitz, en los valles centrales de Oaxaca (Pérez y Anderson, 2013) y que se relaciona también con un cambio hacia condiciones más húmedas en el Holoceno temprano. Tales interpretaciones concuerdan con lo mencionado por Mueller et al. (2012).

A partir de los 7940 AP, el suelo vertic Cambisol, presenta un mejor desarrollo con rasgos vérticos y carbonatos neoformados, indicando con ello una transición de condiciones más secas hacia ambientes fuertemente estacionales. Sugerimos que este cambio está relacionado con una tendencia hacia condiciones más secas en los inicios del Holoceno medio. En Mesoamérica, esta tendencia ha sido documentada en los registros lacustres de la porción central de México, tales como Cuitzeo (Israde et al., 2002), Pátzcuaro (Bradbury, 1997, 2000), Zacapu (Metcalfe, 1995; Ortega et al., 2002) y Etzatlán (Vázquez et al., 2017), donde fuertes oscilaciones de periodos secos se registran entre 6000 - 5000 AP, similar a lo reportado en el Lago Petén Itzá y Chichancanab en la península de Yucatán, alrededor de los 5600 AP (Hodell et al., 2005a, 2008).

Los suelos (calcic Fluvisol) registrados entre 7900 5300 AP, son suelos cumúlicos que se desarrollaron bajo condiciones húmedas con algunas fases secas debido a la concentración y neoformación de carbonatos. Esta tendencia se registra en las secuencias aluviales del valle de Nochixtlán, donde una incisión en el valle (aluviones) cubre un periodo entre los 10000 y 4000 AP.

Otro de los rasgos característicos de los suelos desarrollados a partir de los 7900 AP es la presencia de carbón macroscópico, utilizado como indicador de perturbación antrópica y actividad agrícola (Whitlock y Larsen, 2001; Joyce y Goman, 2012). Esto nos permite sugerir que la transformación del paisaje y el impacto antrópico en los suelos del valle de Yanhuitlán posiblemente inicia desde los 7900 AP; consistente con los fechamientos de restos macroscópicos de Zea Maiz recuperado en Guila Naquitz (Pérez y Anderson, 2013). Estos autores sugieren que las actividades agrícolas y de domesticación del maíz son anteriores a los 6600 AP; edades que han sido documentadas también en diversos sitios arqueológicos tales como en el valle de Tehuacán y en el valle Central del Balsas (Piperno y Flannery, 2001).

Los suelos del Holoceno temprano están poco desarrollados, y han sido interrumpidos por sedimentos aluviales laminados con estratificación cruzada, lo que implica un cambio hacia condiciones más húmedas, como también se ha documentado para las secuencias aluviales estudiadas en el valle de Nochixtlán (Muller et al., 2012), Puebla (Tovar et al., 2013), Tlaxcala (Solís-Castillo et al., 2012), Guanajuato (Borejsza et al., 2008; Borejsza y Frederick, 2010), Tabasco (Solís-Castillo et al., 2014) y Sonora (Cruz-y-Cruz et al., 2015).

\subsection{OCUPACIÓN HUMANA E IMPACTO ANTRÓPICO}

El suelo calcic Vertisol (5300 AP) ha sido reportado por Mueller et al. (2012) como el último de los suelos que fue modificado por agricultura $\mathrm{u}$ otras actividades humanas y que puede observarse bajo uno de los montículos del sitio arqueológico Etlaltongo (3600 AP). Sin embargo, como se ha mencionado, es posible que los grupos humanos se hayan asentado en el valle de Yanhuitlán desde hace aproximadamente $7000 \mathrm{AP}$, a pesar de que la evidencia más temprana de la presencia humana 
en el valle de Yanhuitlán con base en el registro arqueológico, inicia durante el Formativo medio-temprano (1600 - $500 \mathrm{AC}, 3600$ - 2,500 AP), Fase regional Cruz (Spores, 1967).

A partir de los 5300 AP, los suelos de poco desarrollo (vertic Fluvisol) evidencian un aumento en el impacto antrópico en la cubierta edáfica debido a la alta concentración de fragmentos de carbón en la matriz del suelo y la continua interrupción del desarrollo del suelo por sedimentos aluviales. Los suelos desarrollados a los 2730 y 1060 AP (vertic Fluvisol), se relacionan con la estratigrafía cultural, correspondiendo con el Formativo (Fase Cruz) y el Clásico (Fase Las Flores) respectivamente.

Es durante estos periodos culturales que el impacto por las actividades antrópicas principalmente sobre las laderas de las tierras altas promovió la degradación y erosión del suelo. En el periodo Clásico la intensificación del uso de suelo en las pendientes y un aumento en la explotación de los recursos forestales, resultó en un incremento en la capacidad de descarga del río Yanhuitlán y sus afluentes, por lo que la constante acumulación de nuevos sedimentos, el carbón macroscópico y fragmentos de cerámica está presente en los suelos.

Los suelos desarrollados a partir de los 5000 AP están interrumpidos por sedimentos aluviales, estos episodios de inestabilidad en el paisaje, regularmente están relacionados con el incremento de las prácticas agrícolas, aumento en la densidad de población y la construcción de asentamientos humanos. Kirkby (1972) y Mueller et al. (2012) relacionan los eventos erosivos con la intensificación agrícola en Nochixtlán y Río Verde, Goman et al. (2010) añade que el aumento y disminución en la concentración de partículas de carbón en los sedimentos lacustres, está relacionado con los periodos de ocupación y abandono. En Yanhuitlán los sedimentos aluviales producto de los eventos erosivos están relacionados con los periodos de abandono, ya que el contenido de partículas de carbón en los mismos disminuye y a partir de su espesor, es posible establecer periodos de corta duración, mientras que los periodos de formación de suelo (estabilidad) tienen un mayor contenido de frag- mentos de carbón, lo que implica un uso constante del mismo y permite un control de la erosión-sedimentación en los lamabordos.

\subsection{TERRAZAS Y LAMABORDOS}

Los paisajes culturales en la Mixteca Alta están relacionados con las prácticas agrícolas (Figura 5hi). El amplio sistema de terrazas es el resultado de procesos de modificación y aprovechamiento del espacio físico a través del tiempo. Diversos autores han enfocado sus estudios a reconocer las trasformaciones paralelas de la sociedad y el espacio fisico (Butzer, 1980; Criado, 1999; Joyce y Goman, 2012). En la región de Yanhuitlán la construcción de terrazas de fondo de valle (lamabordos) (Figura 5i), corresponde a una técnica agrícola y arquitectónica predominante (Palacio-Prieto et al., 2016; Pérez, 2016).

$\mathrm{El}$ incremento en la capacidad de carga y transporte de sedimentos del drenaje en el valle de Yanhuitlán ha sido propuesto como un detonante para la conformación de terrazas y lamabordos. Kirkby (1972) reconoce que tanto antes como después del desarrollo agrícola en la Mixteca Alta, existía una dinámica significativa de aluvionamiento en el paisaje. Spores (1967) y Kowalewski et al. (2009) sugieren que las terrazas de valle (lamabordos) y las terrazas de contorno vinculadas a sitios arqueológicos del Formativo y el periodo Clásico funcionaron como un medio para mitigar el impacto de la erosión. En el valle de Nochixtlán han sido fechados los lamabordos expuestos por la incisión de los drenajes en posiciones estratigráficas que datan de los periodos Formativo, Clásico y Posclásico (Pérez et al., 2011; Mueller et al., 2012; Leigh et al., 2013). Las edades más tempranas de los lamabordos están asociadas con un paleosuelo datado en 3040 AP (Mueller et al., 2012).

En el valle de Yanhuitlán se ha establecido la edad de los paleosuelos que indican el tiempo de formación de los lamabordos con base en la correlación entre los rasgos pedogenéticos y las edades de radiocarbono. Es importante señalar que las terrazas descritas son de origen antrópico, la primera terraza (TL4, Figura 2c) se desarrolla por 
procesos de erosión y acumulación; en la sección Yan 05 se tiene evidencia de estos procesos, aunque el desarrollo de suelos indica que los patrones de sedimentación cambian abruptamente debido a la presencia de actividades antrópicas (carbones macroscópicos). En este periodo de estabilidad se desarrolla un suelo vertic Cambisol con una edad 7940 AP.

En la secuencia Yan 05 se presenta una nueva fase de actividad, durante la cual se depositan sedimentos que conforman los suelos calcic Fluvisol, caracterizados por un incipiente desarrollo de horizontes A. Estos eventos forman la terraza TL3 (Figura 2c), cuya fase de inestabilidad es con certeza el resultado de las actividades humanas que provocan un aumento en la erosión. Hace 5300 AP, una nueva fase de estabilidad conformó la terraza TL2, sobre la cual se desarrolló un suelo calcic Vertisol y que ha sido localizado bajo el sitio de Etlaltongo (3800 AP); sus rasgos como: el color café obscuro (negro); su morfología de bloques angulares con procesos de expansión-contracción; su compactación y la presencia de restos macroscópicos de carbón, nos permiten identificarlo como un marcador estratigráfico de actividad humana en el valle de Yanhuitlán. Finalmente, una nueva fase de inestabilidad durante el periodo Formativo y Clásico conforman la terraza TL1, en la que el desarrollo de incipientes horizontes A vertic FLUVISOL, con acumulaciones de carbonatos pedogenéticos, registran fases secas durante el periodo Clásico. Los periodos de inestabilidad se presentan con mayor frecuencia a partir de este periodo.

\subsection{EL ANTROPOGENO Y LA ARQUEOLOGÍA EN LA MIXTECA ALTA.}

De acuerdo a los datos obtenidos a partir de este trabajo en la Mixteca Alta ha surgido la siguiente pregunta, ¿`será posible contribuir mediante estas investigaciones con la definición e implicaciones de establecer el término de Antropoceno como una época en la Escala Estratigráfica Internacional (EEI)?, ¿'será factible contribuir a la discusión enfocada al establecimiento de esta época relacionada específicamente con las actividades humanas y sus repercusiones en el sistema Tierra?

El Antropoceno, desde el punto de vista de la Arqueología Ambiental, permite establecer una interacción entre las sociedades humanas y el ambiente. Numerosas investigaciones han reconocido la significativa influencia de los humanos en la Tierra, incluyendo el desencadenamiento del cambio climático (Crutzen y Stoermer, 2000).

Han sido propuestos varios puntos de inicio del Antropoceno con base en diferentes observaciones. Uno de ellos propone al año 2000 DG como punto de inicio de una nueva época (Vitousek et al., 1997; Crutzen, 2002; Crutzen y Steffen, 2003;), que en realidad más que una nueva época lo que se propone es una subdivisión del Holoceno en su punto más actual, es decir, una edad (o piso) dentro del mismo, debido a la corta duración que podría representar. El establecimiento de esta fecha se ha propuesto y relacionado con los esfuerzos por entender y mitigar los efectos humanos en los sistemas globales, es decir, el momento en que la comunidad geocientífica se interesó de una manera más real en el tema. Desde otro enfoque distinto otro investigador ha propuesto como inicio del Antropoceno la Revolución Industrial (entre 1760 y 1830 DG), (Barnosky, 2013), debido al impacto de las emisiones de $\mathrm{CO}_{2}$ en la atmósfera. Sin embargo, esto ha sugerido la idea de que los humanos antes de dicho marcador vivían en armonía con la naturaleza, lo cual en muchos casos ha sido demostrado como incorrecto. Por otro lado, también se ha planteado el establecimiento del dominio político y cultural europeo sobre los pueblos indígenas americanos como una fecha inicial del Antropoceno (1500 DC) (Crosby, 1972, 1986; Thomas, 1989, 1990), debido al elevado intercambio ecológico global, en particular de la flora y fauna, lo que produjo un marcador paleontológico, estratigráfico y cronológico claro a nivel mundial.

A partir de los datos generados para la Mixteca Alta en éste y otros estudios, además de otras regiones como la Zona Maya, región del Balsas, entre otros, se ha observado sin embargo, que los asentamientos humanos ocurrieron desde mucho 
tiempo antes (hace aproximadamente $8000 \mathrm{AP}$ ), los cuales modificaron el entorno al grado de integrar vestigios en el registro estratigráfico (edafosedimentario) regional que persisten hasta nuestros días. Lo anterior nos conduce a una perspectiva diferente relacionada con el planteamiento del inicio de la edad de Antropoceno, ya que los indicadores de actividad humana muestran un fuerte impacto mucho tiempo antes de las fechas propuestas por diversos investigadores hasta el momento. Con base en lo anterior, en el presente trabajo se argumenta en favor de la propuesta relacionada con la inclusión del término Antropoceno dentro de la EEI, sustituyendo en su totalidad la época de Holoceno por este nuevo término de Antropoceno, apoyando los trabajos propuestos por Braje y Erlandson (2013) y Smith y Zeder (2013), donde se presentan argumentos en este mismo sentido.

El término Holoceno, se considera como ambiguo, de acuerdo a las características que se ha visto que están sirviendo de marcadores de esta época; al analizar el sentido de su etimología, que nos lleva a conocer de manera simplista el por qué fue acuñado este término, podemos observar que Holos significa "todo" y Cainos "reciente" es decir su significado primordial está relacionado con todo lo que ocurre en la etapa más reciente de la historia de la Tierra "todo lo reciente"; de acuerdo a los criterios y argumentos mencionados en el presente trabajo, este término efectivamente engloba todo lo que ha ocurrido en los últimos miles de años en la tierra, sin embargo, no diferencia eventos como los ciclos interglaciales previos del Pleistoceno, por lo que tan poco se ha establecido con sumo cuidado todos los eventos climático-ambientales que han ocurrido en la última etapa de la historia de la Tierra, y es evidente que solo coincide con el último ciclo interglacial.

$\mathrm{Al}$ observar similitudes con otros intervalos de la EEI, donde ha sido trascendente la presencia de alguna especie animal o vegetal en particular para el establecimiento de la misma, nos preguntamos ¿Por qué se hace una excepción en este caso y no se permite la consideración de la especie humana como un factor lo suficientemente importante para el establecimiento del nombre de esta época? Desde un punto de vista paleontológico, la especie humana podría cumplir con las características especificadas para un fósil índice en la Tierra, esto significa que se haya expandido por todo el planeta en un intervalo de tiempo muy corto y que el número de individuos sea muy grande. $\mathrm{Al}$ analizar el tiempo de estancia de la especie humana en la Tierra, ésta representa un pequeño pulso de actividad dentro de esta larga historia $(0.00025 \%$ de la edad de la Tierra), lo cual es comparable con diversos fósiles índice que han ocurrido a lo largo de la historia de la misma, además que podríamos atrevernos a sugerir que la especie humana como la conocemos hoy en día, geológicamente hablando podría ser posible que llegue a dominar por un periodo de tiempo relativamente corto en la Tierra.

La EEI está constituida por épocas de muy larga duración, siendo la más corta de todas el Holoceno, lo cual en caso de ser propuesto de acuerdo a otros autores, que el Antropoceno correspondiera a una época más, junto con el Holoceno, resultaría excesiva la subdivisión en dos épocas para éste último intervalo de tiempo tan pequeño, debido a la aún más pequeña duración del Antropoceno. Por otro lado, en caso de considerar a la revolución industrial o la colonización de América como puntos marcadores del Antropoceno, ambas representan características de eventos ocurridos en la Tierra de manera paralela, solo que desde puntos de vista diferentes. Finalmente es importante mencionar que el proponer o discutir si la fecha de inicio del Antropoceno puede ser la etapa de la revolución industrial o un poco antes, resulta poco trascendente, cuando en realidad lo que se intenta discutir en estos momentos es la importancia de los efectos que ha tenido la especie humana sobre la Tierra y si amerita esta característica para ser considera dentro de la EEI como marcador estratigráfico. Cabe señalar que el inicio del Antropoceno está situado espacialmente, es decir, no representa el mismo tiempo en todos lados sobre la superficie terrestre. Con base en todos los argumentos anteriores, el presente trabajo apoya la idea de sustituir 
la época Holoceno en su totalidad por una nueva época denominada Antropoceno más representativa de la especie actualmente dominante en la Tierra y que ha dejado rastros a nivel global desde hace varios milenios.

\section{Conclusiones}

Este estudio pedoestratigráfico analizó los paleosuelos aluviales sepultados y preservados en las terrazas culturales del río Yanhuitlán. Las secuencias de paleosuelos registran los cambios ambientales y su relación con los grupos humanos asentados en la región, con coincidencia cronológica y ambiental de registros en áreas vecinas de Guila Naquitz, Río Verde y el valle de Nochixtlán. En particular los suelos vérticos se asocian con la ocupación del período Formativo temprano, mientras que los suelos dominados por la acumulación de humus están asociados con el periodo Clásico y Posclásico. Los suelos vérticos son los más desarrollados y se interpretan como periodos de estabilidad más larga del paisaje y pedogenésis bajo climas estacionales, mientras que los suelos del periodo Clásico y Posclásico son menos desarrollados y se ven afectados por procesos de erosión y sedimentación, producto de la intensificación de las actividades agrícolas y la degradación del suelo.

Las modificaciones que el hombre ha ejercido se definieron con base en el reconocimiento de la firma antrópica de los suelos culturales. La huella humana en el valle de Yanhuitlán está relacionada con el desarrollo de suelos antrópicos que datan de hace aproximadamente 5000 AP; en estos suelos las actividades humanas dejaron firmas estratigráficas indelebles que son visibles en los registros arqueológicos, geomorfológicos y paleoambientales. La transformación del paisaje producto de la implementación de nuevas tecnologías agrícolas en el valle de Yanhuitlán (lamabordos), es una evidencia de que las sociedades humanas han tenido efectos regionales sobre el paisaje y los ecosistemas que han ocupado desde el Formativo temprano (Holoceno medio).
Los paleosuelos culturales y la construcción de lamabordos en la Mixteca Alta muestran la gran capacidad transformadora del paisaje por el hombre, con etapas de gran acumulación de sedimento derivadas de la degradación ambiental por uso y manejo de las especies vegetales así como el inicio de prácticas agrícolas. Con base en esta información se brinda nuevo sustento relacionado para el debate de la inclusión del Antropoceno dentro de la EEI en sustitución de la época del Holoceno. Lo anterior fundamentado en la correlación de datos estratigráficos y arqueológicos presentes en la región de estudio, más que por los cambios climáticos propuestos por otros autores y que ofrecen mayor incertidumbre.

\section{Agradecimientos}

Este proyecto se realizó gracias al apoyo de los proyectos CONACYT PN247048 y PAPIIT IA107317. Agradecemos el apoyo de Silke Cram, Marco A. Muñoz y Xóchitl Ramírez Miguel, quienes colaboraron en el trabajo de campo. Hilda Rivas brindó apoyo técnico en la realización de los análisis en el Laboratorio de Suelos y Agua del CIGA (Centro de Investigaciones en Geografía Ambiental). Al Dr. Avto Gogitchaishvili del Laboratorio Universitario de Geofisica Ambiental por las facilidades para la medición de la susceptibilidad magnética.

\section{Referencias}

Barnosky, A.D., 2013, Palaeontological Evidence for Defining the Anthropocene: Geological Society, London, Special publications, 395, 149-165.

Beach, T., Dunning, N., Luzzadder-Beach, S., Scarborough, V., 2003, Depression soils in the lowland tropics of Northwestern Belize: anthropogenic and natural origins, en GómezPompa, A., Allen, M., Fedick, S., JiménezOrsonio, J. (eds), The lowland Maya Area: 
Three Millennia at the Human-Wildland Interface, Haworth Press, Binghamton, NY, 139-174.

Beach, T., Luzzadder, S., Terry, R., Dunning, N., Houston, S., Garrison, T., 2011, Carbon isotopic ratios of wetland and terrace soil sequences in the Maya Lowlands of Belize and Guatemala: Catena, 85, 109-118.

Borejsza, A., Rodríguez L.I., Frederick, C.D., Bateman, M.D., 2008, Agricultural slope management and soil erosion at La Laguna, Tlaxcala, Mexico: Journal of Archaeological Science, 35, 1854-1866.

Borejsza, A., Frederick, C., 2010, Fluvial response to Holocene climate change in loworder streams of central Mexico: Journal of Quaternary Science, 25(5), 762-781.

Bradbury, J., 1997, Sources of glacial moisture in Mesoamerica: Quaternary International, 43/44, 97-110.

Bradbury, J., 2000, Limnologic history of Lago de Pátzcuaro, Michoacán, Mexico for the past 48000 years: impacts of climate and man: Palaeogeography, Palaeoclimatology, Palaeoecology, 163, 65-95.

Braje, T., Erlandson, J., 2013, Looking forward, looking back: Humans, anthropogenic change and the Anthropocene: Anthropocene, 4, 116-121.

Brenner, M., Hodell, D., Curtis, J., Rosenmeier, M., Binford, M., Abbott, M., 2001, Abrupt climate change and Pre-Columbian cultural collapse, en Markgraf, V. (ed.), Interhemispheric climate linkages, Academic Press, New York, EEUU, 87-103.

Butzer, K.W., 1980, Holocene alluvial sequences: problems of dating and correlation, en Gullingford, R.A., Davidson, D.A., Lewin, J. (eds.), Timescales in Geomorphology: Wiley, New York, 131-142.

Carleton, W.C., Campbell, D., Collard, M., 2014, A reassessment of the impact of drought cycles on the Classic Maya: Quaternary Science Reviews, 105, 151-161.
Criado, B.F., 1999, Del terreno al espacio: planteamientos y perspectivas para la arqueología del paisaje: España: Universidad de Santiago de Compostela. CAPA: cadernos de arqueoloxía e patrimonio, N. 6, 88 p.

Crosby, A.W., 1972, Columbian Exchange: The Biological and Cultural Consequences of 1492, Praeger Publishers, Westport, Connecticut.

Crosby, A.W., 1986, Ecological Imperialism: The Biological Expansion of Europe 900-1900, Cambridge University Press, Cambridge, Nueva York

Crutzen, P.J., Stoermer, E.F., 2000, The "Anthropocene": Global Change Newsletter, 41, 17-18.

Crutzen, P.J., 2002, The "anthropocene", en Journal de Physique IV (Proceedings), EDP sciences, 12(10) 1-5.

Crutzen, P.J., Steffen, W., 2003, How long have we been in the Anthropocene Era?: Climate Change, 61, 251-257.

Cruz-y-Cruz, T., Sánchez, G., Sedov, S., TerrazasMata, A., Solleiro-Rebolledo, E., TovarLiceaga, R. E., Carpenter, J., 2015, Spatial variability of Late Pleistocene-Early Holocene soil formation and its relation to early human paleoecology in Northwest Mexico: Quaternary International, 365, 135-149.

Dahlin, B.H., Chambers, M.E., Foss, J.E., 1980, Project Acalches: Re-constructing the Natural and Cultural History of a Seasonal Swamp at El Mirador Guatemala; Preliminary Results, en Matheny, R. (ed.), El Mirador, Peten, Guatemala: An Interim Report, Publication 45, New World Archaeological Foundation; Provo, Utah, 37-59.

Dunning, N.P., Luzzadder-Beach, S.L., Beach, T., Jones, J.G., Scarborough, V., Culbert, T.P., 2002, Arising from the Bajos: The Evolution of a Neotropical Landscape and the Rise of Maya Civilization. Annals of the Association of American Geographers, Washington, DC., 92, 267-283. 
Dunning, N., Beach, T., Luzzadder-Beach, S., 2006, Environmental variability among bajos in the southern Maya Lowlands and its implications for ancient Maya civilization and archaeology, en Lucero, L., Fash, B. (eds.), Pre-Columbian Water Management, University of Arizona Press, Chicago, IL., 111-133.

Erlandson, J.M., Rick, T.C., 2010, Archaeology meets marine ecology: the antiquity of maritime cultures and human impacts on marine fisheries and ecosystems: Annual Review of Marine Science, 2, 231-251.

Fedick, S., Flores, M., Sedov, S., Solleiro, R., Mayorga, S., 2008, Adaptation of Maya homegardens by "container gardening" in limestone bedrock cavities: Journal of Ethnobiology, 28, 290-304.

Ferrusquía, I., 1970, Geología del área Tamazulapan-Teposcolula-Yanhuitlán, Mixteca Alta, Estado de Oaxaca. Excursión geológica México-Oaxaca: Sociedad Geológica Mexicana, 97-119.

Flores-Delgadillo, L., Fedick, S.L., SolleiroRebolledo, E., Palacios-Mayorga, S., OrtegaLarrocea, P., Sedov, S., Osuna-Ceja, E., 2011, A sustainable system of a traditional precision agriculture in a Maya homegarden: Soil quality aspects: Soil and Tillage Research, 113(2), 112-120.

García, A.G., 2011, Urbanismo en la Mixteca Alta, Oaxaca: el caso de Naduza-Ñiaxugue en la región de Coixtlahuaca: Ciudad de México, Escuela Nacional de Antropología e Historia, tesis licenciatura, $151 \mathrm{p}$.

García, E., 2004, Modificaciones al Sistema de Clasificación Climática de Köppen: UNAM, México, Serie Libros Instituto de Geografía, $6,90 \mathrm{p}$.

Gill, R., 2000, The Great Maya Droughts: Water, Life, and Death. University of New Mexico Press, Albuquerque 464 p.

Goman, M., Joyce, A., Mueller, R., Paschyn, L., 2010, Multiproxy paleoecological reconstruction of prehistoric land-use history in the western region of the lower Río Verde Valley, Oaxaca, Mexico: The Holocene, 20(5), 761-772.

Goman, M., Arthur, A.J., Raymond G., Mueller, R., 2013, Paleoecological Evidence for Early Agriculture and Forest Clearance in Coastal Oaxaca, en Joyce, A. (ed.), Polity and Ecology in Formative Period Coastal Oaxaca: Louisville, Colorado, Boulder, University Press of Colorado, 43-64.

Haug, G., Günther, D., Peterson, L., Sigman, D., Hughen, K., Aeschlimann, B., 2003, Climate and the collapse of Maya Civilization: Science, 294(14), 1731-1735.

Heredia, E.V.Y., 2007, Cities on Hills: Classic Society in Mesoamerica's Mixteca Alta: Oxford, United Kingdom British Archaeological Reports, International Series, $1728 \mathrm{p}$.

Hodell, D., Brenner, M., Curtis, J., 2005a, Terminal Classic drought in the northern Maya lowlands inferred from multiple sediment cores in Lake Chichancanab (Mexico): Quaternary Science Reviews, 24, 1413-1427.

Hodell, D., Brenner, M., Curtis, J., MedinaGonzalez, R., Ildefonso-Chan, C., AlbornazPat, A., Guilderson, T., 2005b, Climate change on the Yucatan Peninsula during the Little Ice Age: Quaternary Research, 63, 109-121.

Hodell, D.A., Anselmetti, F.S., Ariztegui, D., Brenner, M., Curtis, J.H., Gilli, A., Grzesik, D.A., Guilderson, T.J., Müller, A.D., Bush, M.B., Correa M.A., Escobar, J., Kutterolf, S., 2008, An 85-ka record of climate change in lowland Central America: Quaternary Science Reviews, 27, 1152-1165.

Hunt, T.L., Lipo, G.P., 2009, Revisiting Rapa Nui (EasterIsland) "Ecocide": Pacific Science, 63(4), 601-616.

Israde, A.I., Garduño-Monroy, V.H., Ortega, M.R., 2002, Paleoambiente lacustre del Cuaternario tardío en el centro del lago de Cuitzeo: Hidrobiológica, 12(1), 61-78. 
IUSS Working Group WRB, 2015, World Reference Base for Soil Resources 2014, update 2015, International soil classification system for naming soils and creating legends for soil maps. World Soil Resources Reports No. 106. FAO, Rome.

Joyce, A., Goman, M., 2012, Bridging the theoretical divide in Holocene landscape studies: social andecological approaches to ancient Oaxacan landscapes: Quaternary Science Reviews, 55, 1-22.

Kennett, D.J., Beach, T.P., 2013, Archaeological and Environmental Lessons for the Anthropocene from the Classic Maya Collapse: Anthropocene, 4, 88-100.

Kirch, P.V., 2005, Archaeology and global change: the Holocene record: Annual Review of Environment and Resources, 30(1), 409-440.

Kirkby, M., 1972, The Physical Environment of the Nochixtlan Valley, Oaxaca, Vanderbilt University, Publication in Anthropology 2, Vanderbilt University, Nashville, 59 p.

Kowalewski, S.A., Balkansky, A.K., Stiver, L.R., Walsh, T.J., Pluckhahn, J.F., Chamblee, V.P., Rodríguez, V.Y., Espinoza, H., Smith, C.A., 2009, Origins of the Nuu: Archaeology in the Mixteca Alta, Mexico: University Press of Colorado, Boulder, 516 p.

Leigh, D.S., Kowalewski, S.A., Holdridge, G., 2013, 3400 years of agricultural engineering in Mesoamerica: lama-bordos of the Mixteca Alta, Oaxaca, Mexico: Journal of Archaeological Science, 40(11), 4107-4111.

Leyden, B.W., 2002, Pollen evidence for climatic variability and cultural disturbance in the Maya Lowlands: Ancient Mesoamerica, 13, 85-101.

Liendo, S.R., Solleiro-Rebolledo, E., SolísCastillo, B., Sedov, S., Ortiz, M.A., 2014, Prehispanic Population Dynamics in the western Maya Lowlands: Evaluating resilence and vulnerability in ancient landscape, en Chase, A., Carborough, V. (eds.), Resilence and vulnerability ancient landscape, Transforming Maya Archeology: IHOPE,
Antropological papers of the American Antropological Asociation, Willey Backwell, Hoboken, N.J. 84-101.

Lind, M.D., 2008, Arqueología de la Mixteca: Desacatos, 27, 13-32.

Lozano, G.S., Sosa, N.S., Sugiura, Y., Caballero, M., 2005, 23,000 years of vegetation history of the Upper Lerma, a tropical high-altitude basin in Central Mexico: Quaternary Research, 64, 70-82.

Martiny, B., Martínez-Serrano, R.G., MoránZenteno, D.J., Macías-Romo, C., Ayuso, R.A., 2000, Stratigraphy, geochemistry and tectonic significance of the Oligocene magmatic rocks of western Oaxaca, southern Mexico: Tectonophysics, 318(1), 71-98.

McClung, T.E., 2013, Enfoques ecológicos para la arqueología mexicana: el impacto del hombre en su medio ambiente: Arqueología Mexicana, 121, 66-70.

Medina-Elizalde, M., Polanco-Martínez, J.M., Lases-Hernández, F., Bradley, R., Burns, S., 2016, Testing the "tropical storm" hypothesis of Yucatan Peninsula climate variability during the Maya Terminal Classic Period: Quaternary Research, 86(2), 111-119.

Metcalfe, S., 1995, Holocene environmental change in the Zacapu basin, Mexico: a diatom based record: The Holocene, 5, 196-208.

Mueller, R.G., Joyce, A.A., Borejsza, A., 2012, Alluvial archives of the Nochixtlan valley, Oaxaca, Mexico: age and significance for reconstructions of environmental change: Palaeogeography, Palaeoclimatology, Palaeoecology, 321, 121-136.

Ortega, B., Caballero, C., Lozano, S., Israde, I., Vilaclara, G., 2002, 52000 years of environmental history in Zacapu basin, Michoacán, Mexico: the magnetic record: Earth and Planetary Science Letters, 202, 663-675.

Ortega, B., Vázquez, G., Caballero, M., Israde, I., Lozano, G.S., Schaaf, P., Torres, E., 2010, Late Pleistocene: Holocene record of 
environmental changes in Lake Zirahuen, Central Mexico: Journal of Paleolimnology, 44, 745-760.

Osorio, J., 2015, El sistema-mundo de Wallerstein y su transformación. Una lectura crítica: Argumentos, 28, 77, 131-153.

Palacio-Prieto, J.L., Rosado-González, E., Ramírez-Miguel, X., Oropeza-Orozco, O., Cram-Heydrich, S., Ortiz-Pérez, M.A., Figueroa-Mah-Eng, J.M., Fernández de Castro-Martínez, G., 2016, Erosion, Culture and Geoheritage; the Case of Santo Domingo Yanhutlán, Oaxaca, México: Geoheritage, 8, 359-369.

Pérez, R.V., Anderson, K.C., Neff, M.K., 2011, The Cerro Jazmín Archaeological Project: Investigating Prehispanic Urbanism and Its Environmental Impact in the Mixteca Alta, Oaxaca, Mexico: Journal of Field Archaeology, 36(2), 83-99.

Pérez, R.V., Anderson, K.C., 2013, Terracing in the Mixteca Alta, México: Cycles of resilience of an ancient land use strategy: Human Ecology, 41(3), 335-349.

Pérez, R.V., 2016, Terrace Agriculture in the Mixteca Alta Region, Oaxaca, Mexico: Ethnographic and Archeological Insights on Terrace Construction and Labor Organization Culture: Agriculture, Food and Environment, 38(1), 18-27.

Piperno, D.R., Flannery, K.V., 2001, The earliest archeological maize (Zea mays L.) form highland Mexico: new accelerator mass spectrometry dates and their implications: Proceedings of the Natural Academy Sciences of the United States of America, 98, 2101-2103.

Piperno, D.R., Moreno, J.E., Iriarte, J., Holst, I., Lachniet, M., Jones, J.G., Ranere, A.J., Castanzo, R., 2007, Late Pleistocene and Holocene environmental history of theIguala valley, central Balsas watershed of Mexico: Proceedings of the National Academy of Sciences, 104, 11874-11881.
Plunket, P., 1990, Patrones de asentamiento en el Valle de Nochixtlán y su aportación a la evolución cultural en la Mixteca Alta, en Marcus, W. (ed.), Lecturas históricas del estado de Oaxaca. I. Época prehispánica: Instituto Nacional de Antropología e Historia, México, 349-378.

Redman, G.L., 2004, Effects of agriculture and urban society, en Redman, C.L., James, S.R., Fish, P.R., Rogers, J.D. (eds.), Archaeology of Global Change: The Impacts of Humans on Their Environment (Part 2): Smithsonian Institution Press, Washington, DC, 89-93.

Retallack, G.J., 1990, Soils of the past: an introduction to paleopedology: London, Wiley-Blackwell, second edition, $404 \mathrm{p}$.

Romero, F.M.A., 1996, El sol y la cruz: Los pueblos indios de Oaxaca colonial México, D.F.: Centro de Investigaciones y Estudios Superiores en Antropología Social and Instituto Nacional Indigenista, 291 p.

Rosenmeier, M., Hodell, D., Brenner, M., Curtis, J., Guilderson, T., 2002, A 4000-year lacustrine record of environmental change in the southern Maya lowlands, Petén, Guatemala: Quaternary Research, 57, 183-190.

Sánchez-Pérez, S., Solleiro-Rebolledo, E., Sedov, S., McClung de Tapia, E., Golyeva, A., Prado, B., Ibarra-Morales, E., 2013, The Black San Pablo paleosol of the Teotihuacan Valley, Mexico: pedogenesis, fertility, and use in ancient agricultural and urban systems: Geoarchaeology, 28, 249-267.

Schlaepfer, C., De Pablo, L., 1970, Minerales arcillosos e interpretación sedimentológica de las capas rojas de la formación Yanhuitlán, Oaxaca: Boletín de la Asociación Mexicana de Geólogos Petroleros, XXIII(7-12) 93-117.

Schlaepfer, C., 1976, Geología terciaria del área de Yanhuitlán-Nochixtlán. En: Excursión México-Oaxaca: Servicio Geológico Mexicano, 85-96.

Smith, B.D., Zeder, M.A., 2013, The onset of the Anthropocene: Anthropocene, Elsevier, 4, 8-13. 
Solís-Castillo, B., Solleiro-Rebolledo, E., Sedov, S., Salcido-Berkovich, C., 2012, Paleosuelos en secuencias coluvio-aluviales del Pleistoceno-Holoceno en Tlaxcala: registros paleoambientales del poblamiento temprano en el centro de México: Boletín de la Sociedad Geológica Mexicana, 64(1), 91-108.

Solís-Castillo, B., Solleiro-Rebolledo, E., Sedov, S., Liendo, R., Ortiz-Pérez, M., LópezRivera, S., 2013a, Paleoenvironment and human occupation in the Maya Lowlands of the Usumacinta River, Southern Mexico: Geoarchaeology, 28(3), 268-288.

Solís-Castillo, B., Thiel, C., Cabadas, H., SolleiroRebolledo, E., Sedov, S., Terhorst, B., Damm, B., Frechen, M., Tsukamoto, S., 2013b, Holocene sequences in the Mayan Lowlands: a provenance study using heavy mineral distributions. Eiszeitalter und Gegenwart: Quaternary Science Journal 62 (2), 4-17.

Solís-Castillo, B., Ortiz, M., Solleiro-Rebolledo, E., 2014, Unidades geomorfológico-ambientales de las Tierras Bajas Mayas de TabascoChiapas en el Río Usumacinta: un registro de los procesos aluviales y pedológicos durante el Cuaternario: Boletín de la Sociedad Geológica Mexicana, 66(2), 279-290.

Solís-Castillo, B., Golyeva, A., Sedov, S., SolleiroRebolledo, E., Lopez-Rivera, S., 2015, Phytoliths, stable carbon isotopes and micromorphology of a buried alluvial soil in Southern, Mexico: a polychronous record of environmental change during Middle Holocene: Quaternary International, 365, 150-158.

Spores, R., 1967, The Mixtec Kings and Their People: The Civilization of the American Indian series, 85. Norman: University of Oklahoma Press, 278 p.

Spores, R., 1972, An Archaeological Settlement Survey of the Nochixtlan Valley, Oaxaca: Vanderbilt University, Nashville, 206 p.

Stuiver, M., Reimer, P., 1993, Extended 14G database and revised CALIB radiocarbon calibration program: Radiocarbon, 35, 215-230.

Stuiver, M., Reimer, P.J., Reimer, R., 2016, Calib radiocarbon calibration program, version: CALIB 7.1, Reino Unido: http://calib.qub. ac.uk/calib/calib.html (acceso 10.02.16).

Thomas, D.H., 1989, Archaeological and Historical Perspectives on the Spanish Borderlands West. Columbian Consequences, 1. Smithsonian Institution Press, Washington, DC, $511 \mathrm{p}$.

Thomas, D.H., 1990, Archaeological and Historical Perspectives on the Spanish Borderlands East, Columbian Consequences, 2. Smithsonian Institution Press, Washington, DC, 586 p.

Tovar, R.E., Sedov, S., Solís, B., Solleiro, E., 2013, Dark humic alluvial paleosols in Central and Southern Mexico: micromorphological indicators of Late Pleistocene megafauna habitats: Spanish Journal of Soil Science, 3, 217-235

Tricart, J., Cailleux, A., 1972, Introduction to climatic geomorphology: Longman, London, $235 \mathrm{p}$.

Vázquez, G.G., Solís, B., Solleiro-Rebolledo, E., Goguitchaichvilli, A., Morales, J.J., 2016, Mineral magnetic properties of an alluvial paleosol sequence in the Maya Lowlands: Late Pleistocene-Holocene paleoclimatic implications: Quaternary International, 418, $10-21$.

Vázquez, G., Roy, P., Solis, B., Smith, S., Blanco, E., Lozano-Santacruz, R., 2017, Palaeoenvironment of Etzatlán-Magdalena Basin in western-central Mexico over the Holocene and evaluation of main atmospheric forcings: Palaeogeography, Palaeoclimatology, Palaeoecology, 486, 149-157.

Vitale, L., 1983, Hacia una historia del Ambiente en América Latina: de las culturas aborígenes a la crisis actual. Nueva sociedad/ Editorial Nueva imagen, $70 \mathrm{p}$. 
Vitousek, P.M., Mooney, H.A., Lubchenco, J., Melillo, J.M., 1997, Human domination of Earth's ecosystems: Science, 277(5325), 494-499.

Wallerstein, I.M., 1979, El moderno sistema mundial. La agricultura capitalista y los orígenes de la economía-mundo europea en el siglo xvii, Tomo 1, México, Siglo XX Editores, 640 p.

Whitlock, C., Larsen, C., 2001, Charcoal as a fire Proxy in Tracking Environmental Change Using Lake Sediments, V. 2, en Smol, J.P.,
Burks, H.J.B., Last, W.M. (eds.), Biological Techniques and Indicators Dordrecht, Netherlands. Kluwer Academic Publishers, 75-97.

Williams, E., 2004, Nuevas perspectivas sobre el sistema mundial mesoamericano. Relaciones, 99, 25, 145-173.

Zarate, R., 1987. Excavaciones de un sitio Preclásico en San Mateo Etlatongo Nochixtlán, Oaxaca, México: British Archaeological Reports, International Series, 322, Oxford, $138 \mathrm{p}$. 